\title{
ABHYANKAR'S WORK ON YOUNG TABLEAUX AND SOME RECENT DEVELOPMENTS
}

\author{
SUDHIR R. GHORPADE
}

Dedicated to Professor Shreeram Abhyankar on his sixtieth birthday

\section{Contents}

1. Introduction

2. Preliminaries plus Preview

3. Enumeration of Standard Tableaux

4. Universal Determinantal Identity

5. Indexed Monomials

6. Determinantal Ideals and their Hilbert Functions

7. Some Recent Developments

7.1. Enumeration of Standard Multitableaux

7.2. Straightening Law in Higher Dimensions

7.3. Bijection between Standard Bitableaux and Indexed Monomials 24

7.4. Standard Multitableaux and Monomials 25

7.5. Ladder Determinantal Varieties 25

7.6. Coefficients of the Hilbert Function of $I(p, a) \quad 25$

References

\section{INTRODUCTION}

This paper is meant to be a survey of Abhyankar's work on Young tableaux and some of the subsequent developments motivated by it. Leaving the technical details to the remaining sections, we now attempt to give an overview by narrating some background, a little bit of history, and some stories in the two paragraphs below. In keeping with the spirit of Abhyankar's work on Young tableaux, much of this paper is of an elementary nature except perhaps for a few words thrown here and there.

The initial motivation for Abhyankar's work on Young tableaux came from a discussion with V. Deodhar in 1982 following which he investigated the singularities of Schubert varieties in flag manifolds, and showed that for a class of such varieties, the tangent cone at the so called distinguished point is given by an ideal of the type $I_{p}(L)$, the ideal generated by $p \times p$ minors in $L$ where $L$ is a ladder shaped subset of a rectangular matrix with indeterminate entries. This led him to study such determinantal ideals in general. The combinatorial notion of Young tableaux was invented by Alfred Young in 1902 [Y] for its applications to invariant theory and the representation theory of the symmetric group. About 70 years later, DoubiletRota-Stein [DRS] obtained the remarkable Straightening Law (or Standard Basis Theorem) using a two-sided variant of Young tableaux, called (Young) bitableaux.

Date: August 9, 1991.

This is an unofficial electronic version of the paper which has appeared in Algebraic Geometry and its Applications (West Lafayette, IN, 1990), C. Bajaj Ed., Springer-Verlag, New York, (1994), pp. 233-265; MR 95h:05148. Some of the references have been updated. 
This result, which we shall soon describe in detail, was clearly seen to be of great importance for the study of determinantal varieties and numerous applications in this direction were soon given by DeConcini-Eisenbud-Procesi [DEP]. Learning of these developments, Abhyankar began the project of "understanding" it all in his own inimitable manner. By 1983, he had a new proof of the Straightening Law using enumerative techniques. This was a remarkable display of tour de force, and all the combinatorial results were independently proved only from the basic principles. The multitude of enumerative formulas Abhyankar obtained, and the implied insight appears to be incredible. At any rate, that's how it seemed to me when I would travel to Bombay University to attend the series of lectures by Professor Abhyankar on this topic while I was doing my M.Sc. at I.I.T. Bombay. I might add that what attracted me to begin attending these lectures was the little note in the seminar notice saying that only High School Algebra background would suffice to understand these lectures! Now apart from being of an interest in itself, the enumeration has its advantages. In short, counting counts. Indeed, it turns out that Abhyankar's counting yields explicit formulas for the Hilbert functions of a class of determinantal ideals which includes the ideal generated by $p \times p$ minors of a matrix with indeterminate entries. The Hilbert functions are in fact polynomials for all parameter values in the case of these ideals. Primality of these ideals, and in fact, of a more general class of ideals, which include the ladder determinantal ideals mentioned earlier, can be deduced from the Standard Basis Theorem (=SBT). Now to prove the SBT itself, the enumeration helps in reducing the work at least by half. The other half is proved using the so called Universal Determinantal Identity which generalizes the quadratic relations satisfied by the Plucker coordinates in Grassmann variety. Following the counting of standard bitableaux and its applications to enumerative proof of SBT and explicit computations of Hilbert functions of determinantal ideals, Abhyankar observed that a certain set of the so called indexed monomials has the same cardinality as the set of standard bitableaux under consideration. This result makes it possible to give an explicit basis for the homogeneous coordinate ring of the ladder determinantal varieties indicated earlier, and also to prove their irreducibility.

When Abhyankar wrote all these results, it became a paper of about 500 pages, and so it came out as a book [A3]. One of the main aims of this paper is to give a short and motivated account of several of Abhyankar's ideas on Young tableaux, thereby hoping to make [A3] more accessible. This paper differs from [A2], which gives a summary of [A3] but no proofs, in the respect that most sections, i.e., except 5 and 7, contain fairly self- contained proofs. In Sections 2 to 6 below, we describe the work of Abhyankar, and in the last section, we discuss some problems suggested by this work, and their status.

\section{Preliminaries plus Preview}

In this section we set some notation, define Young tableaux and formally state the Standard Basis Theorem (=SBT) and give a preview of the succeeding material by way of motivating Abhyankar's strategy for an enumerative proof of the SBT.

Given any two integers $A$ and $B$, by $[A, B]$ we denote the set of integers between $A$ and $B$, i.e., $[A, B]=\{C \in \mathbb{Z}: A \leq C \leq B\}$. Let $q$ be a positive integer. Given a positive integer $p$, a $q$-sided strictly increasing sequence of positive integers such that the length of each side is $p$, i.e., a map $a:[1, q] \times[1, p] \rightarrow \mathbb{Z}$ such that $1 \leq a(k, 1)<\cdots<a(k, p)$ for $k=1, \ldots, q$, is called a multivector of width $q$ and length $p$; if $q=1$, then $a$ is called a univector, whereas if $q=2$, then a is called a bivector. By a (Young) tableau of width $q$ we mean a finite sequence $T=(T[1], T[2], \ldots, T[d])$ of multivectors of width $q$ (and varying length); here the 
nonnegative integer $d$ is called the depth of the tableau $T$, and it may be denoted by $\operatorname{dep}(T)$ (empty tableau being the only one of depth 0 ). Tableaux of width 1 and 2 are called unitableaux and bitableaux respectively, whereas those of higher width are generally referred to as multitableaux. Thus a bitableau $T$ of depth $d$ may be visualized as an array of the following type:

\begin{tabular}{ccc|ccc}
\hline$T[1]\left(1, p_{1}\right)>$ & $\cdots$ & $>T[1](1,1)$ & $T[1](2,1)<$ & $\cdots$ & $<T[1]\left(2, p_{1}\right)$ \\
$T[2]\left(1, p_{2}\right)>$ & $\cdots$ & $>T[2](1,1)$ & $T[2](2,1)<$ & $\cdots$ & $<T[2]\left(2, p_{2}\right)$ \\
& & $\vdots$ & $\vdots$ & & \\
$T[d]\left(1, p_{d}\right)>$ & $\cdots$ & $>T[d](1,1)$ & $T[d](2,1)<$ & $\cdots$ & $<T[d]\left(2, p_{d}\right)$
\end{tabular}

Here we have let $p_{1}, p_{2}, \ldots, p_{d}$ denote the lengths of $T[1], T[2], \ldots, T[d]$ respectively. If we erase one of the sides in the above array, then we "see" a unitableau. To "see" a multitableau of width greater than 2, one can imagine pages of a book opened up so that any two pages are at some distance from each other; the book evidently has $q$ pages. The sum of row lengths of a tableau, which is also the number of elements on (any) one of its sides, is called its area; thus for a tableau $T$ as above, the area equals $p_{1}+p_{2}+\cdots+p_{d}$. Given $q$ positive integers $m(1), \ldots, m(q)$, we say that a tableau $T$ is bounded by $m=(m(1), \ldots, m(q))$ if the entries on the $k$-th side are no larger than $m(k)$, i.e., for each $k \in[1, q]$ we have $T[e](k, i) \leq m(k)$ for all permissible values of $e$ and $i$. One more definition. A tableau $T$ is said to be standard if the row lengths decrease and there is an increase along each column, i.e., in the above notation we have $p_{1} \geq p_{2} \geq \cdots \geq p_{d}$ and for each $e \in[1, d-1]$ we have $T[e](k, i) \leq T[e+1](k, i)$ for $1 \leq k \leq q$ and $1 \leq i \leq p_{e+1}$. The condition of standardness may be briefly written as $T[1] \leq T[2] \leq \cdots \leq T[d]$ where for two multivectors $a[1]$ and $a[2]$ of width $q$ and lengths $p_{1}$ and $p_{2}$ respectively, we set $a[1] \leq a[2]$ iff $p_{1} \geq p_{2}$ and $a[1](k, i) \leq a[2](k, i)$ for all $k \in[1, q]$ and $i \in\left[1, p_{2}\right]$; note that the latter defines a partial order on the set of all multivectors of width $q$. The set of all standard tableaux of width $q$ which are bounded by $m$ will be denoted by $\operatorname{stab}(q, m)$, and given a nonnegative integer $V$, the subset of $\operatorname{stab}(q, m)$ consisting of standard tableaux of area $V$ will be denoted by $\operatorname{stab}(q, m, V)$.

Now that we know what Young tableaux are, one might ask what are they good for. We cite two possible reasons and of these, the second one leads us to motivate the contents of some of the succeeding sections.

The irreducible representations of the symmetric group $S_{n}$ of degree $n$, being equinumerous with the conjugacy classes in $S_{n}$, are in one-to-one correspondence with the partitions of $n$. Given a partition $\lambda=\left(p_{1}, p_{2}, \ldots, p_{d}\right)$ of $n$ [so that $p_{1} \geq$ $p_{2} \geq \cdots \geq p_{d} \geq 1$ and $\left.p_{1}+p_{2}+\cdots+p_{d}=n\right]$, the corresponding irreducible $S_{n}$-module is denoted by $S^{\lambda}$ and is called the Specht module corresponding to $\lambda$. It turns out that the degree of the irreducible representation corresponding to $\lambda$, i.e., $\operatorname{dim} S^{\lambda}$, is exactly equal to the number of standard unitableaux of depth $d$ and row lengths $p_{1}, p_{2}, \ldots, p_{d}$ in which every integer between 1 and $n$ occurs exactly once (or equivalently, they are bounded by $n$ and are "strictly standard" in the sense that they are standard and there is strict increase along the columns as well. Actually this is what A. Young meant by standard). Furthermore, the character table of $S_{n}$ can also be constructed using these tableaux. Indeed, we more or less know everything about representations of $S_{n}$ - thanks to tableaux. For details concerning these results, see G.D. James's monograph [J], or the forthcoming book of B. Sagan [S]. More about the connection of Young tableaux with Representation theory, Invariant theory, and Combinatorics, can be found in $[\mathrm{Ku}]$ and $[\mathrm{St}]$.

To see another domain of applicability, let $X$ be an $m(1) \times m(2)$ matrix whose entries $X_{i j}$ 's are independent indeterminates over some field $K$, and let $K[X]$ denote the ring of polynomials in these $m(1) m(2)$ variables with coefficients in $K$. Let $p$ be a positive integer such that $p \leq \min \{m(1), m(2)\}$. Note that a $p \times p$ minor of $X$ 
is uniquely determined once we prescribe the row and column indices, and thus by a bivector bounded by $m=(m(1), m(2))$ and of length $p$. A bitableau $T$ bounded by $m$ can be made to correspond to the product of the corresponding minors, or in other words, to a monomial in the minors of $X$; it is a homogeneous polynomial whose degree equals the area of $T$. Now we can state the Standard Basis Theorem or the Straightening Law of Doubilet-Rota-Stein. It says that the monomials in the minors of $X$ corresponding to standard bitableaux bounded by $m=(m(1), m(2))$ form a $K$-vector space basis of $K[X]$.

The above basis is quite different from the usual basis (of monomials in $X_{i j}$ 's) and is more useful when one wants to study determinantal ideals and such. The idea of Abhyankar's enumerative proof of SBT is as follows. Fix a nonnegative integer $V$ and consider the $V^{\text {th }}$ homogeneous component $K[X]_{V}$ of $K[X]$. It suffices to show that the monomials in the minors of $X$ corresponding to standard bitableaux bounded by $m$ and of area $V$ form a $K$-vector space basis of $K[X]_{V}$ (for any given $V)$. But now the advantage is that $K[X]_{V}$ is a finite dimensional vector space and we also know its dimension; indeed

$$
\begin{aligned}
\operatorname{dim}_{K} K[X]_{V} & =\text { number of monomials of degree } V \text { in } m(1) m(2) \text { variables } \\
& =\left(\begin{array}{c}
V+m(1) m(2)-1 \\
m(1) m(2)-1
\end{array}\right) .
\end{aligned}
$$

So all we need to do is to show that the cardinality of the set $\operatorname{stab}(2, m, V)$ of all standard bitableaux bounded by $m$ and of area $V$ equals $\operatorname{dim}_{K} K[X]_{V}$, and to prove that the corresponding monomials in the minors of $X$ generate $K[X]_{V}$ or that they are linearly independent. Abhyankar proves the generation part by using the Laplace expansion for suitable determinants; these ideas are essentially similar to those of Hodge in his proof of the Basis Theorem for Grassmann varieties [Ho], and also to those in [DRS] or [DEP]. The problem of finding $|\operatorname{stab}(2, m, V)|$ appears formidable and to tackle this Abhyankar generalizes the set by throwing in some more parameters $p$ and $a$. By giving particular values to these new parameters we get back the set $\operatorname{stab}(2, m, V)$. In greater detail, we fix a positive integer $p$ and a bivector $a$ bounded by $m$ and of length $p$, and consider the set $\operatorname{stab}(2, m, p, a, V)$ which, by definition, consists of all standard bitableaux $T$ bounded by $m$, dominated by $a$, and of area $V$, where a bitableau $T$ is said to be dominated by the bivector $a$ if the tableau obtained by placing $a$ on the top of $T$ is also standard, i.e., $a \leq T[e]$ for all $e \in[1, \operatorname{dep}(T)]$. More generally, we can consider $a$ to be a multivector of width $q$ and define the $\operatorname{set} \operatorname{stab}(q, m, p, a, V)$ in a completely analogous manner. The thought of "monomials in minors" corresponding to multitableaux is rather intriguing, but we shall come to that in a later section. Right now we just regard multitableaux as combinatorial objects waiting to be counted. As to how Abhyankar enumerates this set, we see that in the next section.

\section{Enumeration of Standard Tableaux}

Let us fix positive integers $q, p$, a $q$-tuple $m=(m(1), \ldots, m(q))$ of positive integers, a multivector $a$ of width $q$ and length $p$ such that $a$ is bounded by $m$, and a nonnegative integer $V$. We wish to count the set

$$
\operatorname{stab}(q, m, p, a, V)=\{T \in \operatorname{stab}(q, m): a \leq T \text { and the area of } T \text { is } V\}
$$

where we have written $a \leq T$ to briefly indicate that $T$ is dominated by $a$, i.e., $a \leq T[e]$ for all $e \in[1, \operatorname{dep}(T)]$; note that when $V=0$, this set consists only of the empty tableau.

The first step is to decompose this set into disjoint subsets which are easier to handle. To this effect, we define the shape of a tableau $T \in \operatorname{stab}(q, m, p, a, V)$ to be 
the $p$-tuple $v=(v(1), \ldots, v(p))$ where

$$
\begin{aligned}
v(i) & =\text { the number of rows of length } i \\
& =\mid\{e \in[1, \operatorname{dep}(T)]: \text { the length of } T[e] \text { is } i\} \mid ;
\end{aligned}
$$

we clearly have $v(1)+2 v(2)+\cdots+p v(p)=V$. Given a $p$-tuple $v=(v(1), \ldots, v(p))$ of nonnegative integers, we define

$$
\operatorname{stas}(q, m, p, a, v)=\{T \in \operatorname{stab}(q, m, p, a, V): \text { the shape of } T \text { is } v\} .
$$

Obviously

$$
|\operatorname{stab}(q, m, p, a, V)|=\sum_{v}|\operatorname{stas}(q, m, p, a, v)|
$$

where the sum is taken over all $p$-tuples $v=(v(1), \ldots, v(p))$ of nonnegative integers such that $v(1)+2 v(2)+\cdots+p v(p)=V$. The next reduction is to note that we clearly have

$$
|\operatorname{stas}(q, m, p, a, v)|=\prod_{k=1}^{q}\left|\operatorname{stas}\left(1, m(k), p, a^{(k)}, v\right)\right|
$$

where for $k \in[1, q], a^{(k)}$ denotes the univector of length $p$ obtained from $a$ by restricting to its $k^{\text {th }}$ side so that $a^{(k)}(1, i)=a(k, i)$ for $1 \leq i \leq p$; evidently $a^{(k)}$ is bounded by $m(k)$. So it suffices to consider unitableaux.

Now let us fix a positive integer $\mu$ [to serve as a "bound" for unitableaux], and a $p$-tuple $v=(v(1), \ldots, v(p))$ of nonnegative integers. Let $\alpha, \beta$ denote univectors bounded by $\mu$ and of length $p$. If $\beta \geq \alpha$, then any $T \in \operatorname{stas}(1, \mu, p, \beta, v)$ can be preaugmented by $\beta$ to give a unitableaux $T^{*} \in \operatorname{stas}\left(1, \mu, p, \alpha, v^{*}\right)$, where the "new shape" $v^{*}=\left(v^{*}(1), \ldots, v^{*}(p)\right)$ is given by

$$
v^{*}(i)= \begin{cases}v(i) & \text { if } 1 \leq i \leq p-1 \\ v(p)+1 & \text { if } i=p\end{cases}
$$

and the new unitableaux $T^{*}$ is given by $T^{*}[1]=\beta, T^{*}[e]=T[e-1]$ for $e=$ $2, \ldots, \operatorname{dep}\left(T^{*}\right)=\operatorname{dep}(T)+1$. Conversely, any $T^{*}$ in $\operatorname{stas}\left(1, \mu, p, \alpha, v^{*}\right)$ can be obtained in this way [note that $v^{*}$ is characterized by the property that $v^{*}(p) \neq 0$ ]. It follows that we have the recurrence relation

$$
\left|\operatorname{stas}\left(1, \mu, p, \alpha, v^{*}\right)\right|=\sum_{\beta \geq \alpha}|\operatorname{stas}(1, \mu, p, \beta, v)| .
$$

Furthermore, we clearly have that

(2) if $p \neq 1$ and $v(p)=0$, then $|\operatorname{stas}(1, \mu, p, \alpha, v)|=|\operatorname{stas}(1, \mu, p-1, \tilde{\alpha}, \hat{v})|$

where $\tilde{\alpha}$ is a univector of length $p-1$ and $\hat{v}$ is a $(p-1)$-tuple, obtained by restricting $\alpha$ and $v$ respectively, i.e., $\tilde{\alpha}(1, i)=\alpha(1, i)$ and $\hat{v}(i)=v(i)$ for $1 \leq i \leq p-1$. Lastly, it is clear that

$$
\text { if } p=1 \text { and } v(p)=0 \text {, then }|\operatorname{stas}(1, \mu, p, \alpha, v)|=1 \text {. }
$$

Now we seek a function of the parameters $(\mu, p, \alpha, v)$ satisfying the above relations. After some experimentation, Abhyankar arrived at the function $\nu$ defined as follows.

$$
\nu(\mu, p, \alpha, v)=\operatorname{det}\left(\left[\begin{array}{c}
\mu-\alpha(1, j) \\
v(i)+v(i+1)+\cdots+v(p)+j-i
\end{array}\right]\right)
$$

where the entries of the above $p \times p$ determinant are the so called twisted binomial coefficients which are defined as follows.

$$
\left[\begin{array}{c}
U \\
A
\end{array}\right]=\left(\begin{array}{c}
U+A \\
A
\end{array}\right)= \begin{cases}\frac{(U+1) \cdots(U+A)}{A !} & \text { if } A \geq 0 \\
0 & \text { if } A<0\end{cases}
$$


where $A$ is an integer and $U$ can be any element in an overring of $\mathbb{Q}$, in particular, it can be an indeterminate over $\mathbb{Q}$ in which case the binomial coefficient is a univariate polynomial with rational coefficients. These twisted binomial coefficients are convenient for our purposes and will often appear in this paper. At this point we would also like to remark on the convention that unless mentioned otherwise, all matrices will be of order $p$, and whenever we write $\operatorname{det}\left(a_{i j}\right)$, it may be tacitly assumed that the corresponding matrix is of order $p$ so that the indices $i$ and $j$ vary between 1 and $p$.

To verify (1), let us write $\bar{\alpha}_{j}=\mu-\alpha(1, j), \bar{\beta}_{j}=\mu-\beta(1, j)$, and $\bar{v}(j)=$ $v(j)+v(j+1)+\cdots+v(p)$ for $1 \leq j \leq p$. It is readily seen that

$$
\sum_{\beta \geq \alpha} \nu(\mu, p, \beta, v)=\sum_{0 \leq \bar{\beta}_{p} \leq \bar{\alpha}_{p}} \sum_{\bar{\beta}_{p}<\bar{\beta}_{p-1} \leq \bar{\alpha}_{p-1}} \cdots \sum_{\bar{\beta}_{2}<\bar{\beta}_{1} \leq \bar{\alpha}_{1}} \operatorname{det}\left(\left[\begin{array}{c}
\bar{\beta}_{j} \\
\bar{v}(i)+j-i
\end{array}\right]\right) .
$$

In the first sum, only the first column of the above determinant varies, and so by using the multilinearity of determinants, we can eliminate the first sum. Now we need the following

General Progression Lemma (3.1). Given integers $e, x, y$ with $x<y$, we have

$$
\sum_{x<s \leq y}\left[\begin{array}{l}
s \\
e
\end{array}\right]=\left[\begin{array}{c}
y \\
e+1
\end{array}\right]-\left[\begin{array}{c}
x \\
e+1
\end{array}\right]
$$

This lemma can be proved by induction on $y-x$ using the Basic Binomial Identity

$$
\left[\begin{array}{l}
U \\
A
\end{array}\right]=\left[\begin{array}{c}
U-1 \\
A
\end{array}\right]+\left[\begin{array}{c}
U \\
A-1
\end{array}\right]
$$

whose proof is straightforward. The General Progression Lemma can be applied to each entry in the first column of the resulting determinant, which yields the difference of two determinants of which the first one has $\bar{\beta}_{1}$ replaced by $\bar{\alpha}_{1}$ and $\bar{v}(i)$ by $\bar{v}(i)+1$ in the first column, and the second one vanishes because two of its columns are identical. Continuing in this manner, we see that

$$
\sum_{\beta \geq \alpha} \nu(\mu, p, \beta, v)=\operatorname{det}\left(\left[\begin{array}{c}
\bar{\alpha}_{j} \\
\bar{v}(i)+1+j-i
\end{array}\right]\right)=\nu\left(\mu, p, \alpha, v^{*}\right)
$$

which shows that the function $\nu$ satisfies a relation of the form (1). It is easy to see that $\nu$ also satisfies relations of the form (2) and (3). Hence by (double) induction on $p$ and $v(p)$, it follows that

$$
|\operatorname{stab}(q, m, p, a, V)|=F^{(8)}(q, m, p, a, V)
$$

where we define

$$
F^{(8)}(q, m, p, a, V)=\sum_{v} \prod_{k=1}^{q} \operatorname{det}\left(\left[\begin{array}{c}
m(k)-a(k, j) \\
v(i)+v(i+1)+\cdots+v(p)+j-i
\end{array}\right]\right)
$$

where the sum is taken over all $p$-tuples $v=(v(1), \ldots, v(p))$ of nonnegative integers such that $v(1)+2 v(2)+\cdots+p v(p)=V$.

Now we shall make transformations to obtain different versions of the above formula. One of the motivations in doing so is to be able to deduce that by giving particular values to the parameters $q, p$ and $a$, we have $|\operatorname{stab}(2, m, V)|=\operatorname{dim}_{K} K[X]_{V}$. To this end, we begin by noting that the transformation

$$
u(i)=v(i)+v(i+1)+\cdots+v(p)+p-i, \quad i=1,2, \ldots, p
$$

maps the set of all $p$-tuples $v=(v(1), \ldots, v(p))$ of nonnegative integers with $v(1)+$ $2 v(2)+\cdots+p v(p)=V$ bijectively onto the set of all $p$-tuples $u=(u(1), \ldots, u(p))$ 
of nonnegative integers with $u(1)>u(2)>\cdots>u(p)$ and $u(1)+\cdots+u(p)=$ $V+\frac{p(p-1)}{2}$. Hence if we let

$$
F^{(7)}(q, m, p, a, V)=\sum_{u} H^{(7)}(q, m, p, a, u)
$$

where the sum is taken over all $p$-tuples $u$ with the above properties, and where for any $p$-tuple $u=(u(1), \ldots, u(p))$ of integers we have put

$$
H^{(7)}(q, m, p, a, u)=\prod_{k=1}^{q} \operatorname{det}\left(\left[\begin{array}{c}
m(k)-a(k, j) \\
u(i)+j-p
\end{array}\right]\right),
$$

then we have: $F^{(8)}(q, m, p, a, V)=F^{(7)}(q, m, p, a, V)$.

We will now make further transformations with the assumption that $q$ is even. First of all, we will try to get rid of the clumsy restriction $u(1)>u(2)>\cdots>$ $u(p) \geq 0$ on the parameter $u$ in the above summation. Let us begin by introducing some

Notation. Let $\mathbb{Z}(p)$ denote the set of all integer $p$-tuples, i.e.,

$$
\mathbb{Z}(p)=\{v=(v(1), \ldots, v(p)): v(i) \in \mathbb{Z} \text { for } 1 \leq i \leq p\}
$$

and for any $U \in \mathbb{Z}$, let

$$
\mathbb{Z}(p, U)=\{v \in \mathbb{Z}(p): v(1)+\cdots+v(p)=U\} .
$$

Let $u=(u(1), \ldots, u(p))$ be any element of $\mathbb{Z}(p)$. Observe that we clearly have the following.

(i): If $u(p)<0$ then $u(p)+j-p<0$ for all $j \in[1, p]$, and therefore in this case we have $H^{(7)}(q, m, p, a, u)=0$.

(ii): If $u(i)=u\left(i^{\prime}\right)$ for some $i \neq i^{\prime}$ in $[1, p]$, then $H^{(7)}(q, m, p, a, u)=0$.

(iii): Let $\tau \in S_{p}$ be any permutation of $[1, p]$ and let $u[\tau] \in \mathbb{Z}(p)$ denote the permuted $p$-tuple, i.e., $u[\tau]=(u(\tau(1)), \ldots, u(\tau(p)))$. Then

$$
H^{(7)}(q, m, p, a, u[\tau])=[\operatorname{sgn}(\tau)]^{q} H^{(7)}(q, m, p, a, u)=H^{(7)}(q, m, p, a, u) .
$$

Now given any $p$-tuple $u=(u(1), \ldots, u(p))$ of distinct integers, there exists a unique permutation $\tau \in S_{p}$ such that $u(\tau(1))>u(\tau(2))>\cdots>u(\tau(p))$. Hence, in view of the observations (i)-(iii), we see that $F^{(7)}(q, m, p, a, V)=F^{(7 *)}(q, m, p, a, V)$ where

$$
F^{(7 *)}(q, m, p, a, V)=\frac{1}{p !} \sum_{u \in \mathbb{Z}\left(p, V+\frac{p(p-1)}{2}\right)} H^{(7)}(q, m, p, a, u)
$$

and note that the above summation is essentially finite, i.e., all except finitely many summands are zero. The advantage of letting the parameters $u(1), \ldots, u(p)$ take all integer values is that it facilitates further transformations. For example, the equations

$$
v(i)=u(i)+i-p, \quad i=1,2, \ldots, p
$$

determine a bijective transformation of $\mathbb{Z}\left(p, V+\frac{p(p-1)}{2}\right)$ onto $\mathbb{Z}(p, V)$. Thus if we let

$$
F^{(6)}(q, m, p, a, V)=\frac{1}{p !} \sum_{v \in \mathbb{Z}(p, V)} \prod_{k=1}^{q} G^{(6 k)}(q, m, p, a, v)
$$

where for any $v \in \mathbb{Z}(p)$ and $k \in[1, q]$ we have put

$$
G^{(6 k)}(q, m, p, a, v)=\operatorname{det}\left(\left[\begin{array}{c}
m(k)-a(k, j) \\
v(i)+j-i
\end{array}\right]\right),
$$

then we have: $F^{(7)}(q, m, p, a, V)=F^{(6)}(q, m, p, a, V)$. 
Remark: It may be interesting to note that if $q$ is odd, and $p>1$ then, in view of (i), (ii), (iii) above, we have that $F^{(7 *)}(q, m, p, a, V)=F^{(6)}(q, m, p, a, V)=0$. For a direct proof of this identity, see [Gh1].

Our next step is to get rid of the factor $\frac{1}{p !}$ in $F^{(6)}$ by showing that one of the determinants, say $G^{(6 k)}$, can be pulled out of the product and replaced by $p$ ! times the product of entries on its principal diagonal. We start with the following elementary observations.

(a): If $\left(a_{i j}\right)$ is a $p \times p$ matrix and $\tau \in S_{p}$ is a permutation, then $\operatorname{det}\left(a_{i j}\right)=$ $\operatorname{sgn}(\tau) \operatorname{det}\left(a_{i \tau(j)}\right)$.

(b): Let $f: \mathbb{Z}(p) \rightarrow \mathbb{Q}$ be any map such that $\{v \in \mathbb{Z}(p): f(v) \neq 0\}$ is finite, and let $\sigma \in S_{p}$ be any permutation. Then

$$
\sum_{v \in \mathbb{Z}(p, V)} f(v(1), \ldots, v(p))=\sum_{v \in \mathbb{Z}(p, V)} f(v(\sigma(1)), \ldots, v(\sigma(p))) .
$$

(c): Let $f$ be as in (b), and let any $U \in \mathbb{Z}$ and $u \in \mathbb{Z}(p, U)$ be given. Then

$$
\sum_{v \in \mathbb{Z}(p, V)} f(v(1)+u(1), \ldots, v(p)+u(p))=\sum_{v \in \mathbb{Z}(p, V+U)} f(v(1), \ldots, v(p)) .
$$

Now let us fix some $k \in[1, q]$. Given any $v \in \mathbb{Z}(p, V)$, let us expand the $k^{\text {th }}$ determinant in $F^{(6)}$ to get

$$
G^{(6 k)}(q, m, p, a, v)=\sum_{\tau \in S_{p}} \operatorname{sgn}(\tau) \prod_{j=1}^{p}\left[\begin{array}{c}
m(k)-a(k, j) \\
v(\tau(j))+j-\tau(j)
\end{array}\right]
$$

also let us take the transpose of the remaining matrices and use (a) to write

$$
\prod_{\substack{1 \leq n \leq q \\
n \neq k}} G^{(6 n)}(q, m, p, a, v)=[\operatorname{sgn}(\tau)]^{q-1} \prod_{\substack{1 \leq n \leq q \\
n \neq k}} \operatorname{det}\left(\left[\begin{array}{c}
m(n)-a(n, i) \\
v(\tau(j))+i-\tau(j)
\end{array}\right]\right) .
$$

Now substitute the expressions on the right in the corresponding summand in $F^{(6)}$, interchange the summations, and observe that $[\operatorname{sgn}(\tau)]^{q}=1$. This shows that $p ! F^{(6)}(q, m, p, a, V)$ is equal to

$$
\begin{aligned}
& \sum_{\tau \in S_{p}} \sum_{v \in \mathbb{Z}(p, V)}\left(\prod_{j=1}^{p}\left[\begin{array}{c}
m(k)-a(k, j) \\
v(\tau(j))+j-\tau(j)
\end{array}\right]\right) \prod_{\substack{1 \leq n \leq q \\
n \neq k}} \operatorname{det}\left(\left[\begin{array}{c}
m(n)-a(n, i) \\
v(\tau(j))+i-\tau(j)
\end{array}\right]\right) \\
& =\sum_{\tau \in S_{p}} \sum_{v \in \mathbb{Z}(p, V)}\left(\prod_{j=1}^{p}\left[\begin{array}{c}
m(k)-a(k, j) \\
v(j)+j-\tau(j)
\end{array}\right]\right) \prod_{\substack{1 \leq n \leq q \\
n \neq k}} \operatorname{det}\left(\left[\begin{array}{l}
m(n)-a(n, i) \\
v(j)+i-\tau(j)
\end{array}\right]\right)
\end{aligned}
$$

where the last equality follows from (b). Finally, we use (c) with $u(j)=j-\tau(j)$ to conclude that the above expression is equal to $p ! F^{(5 k)}(q, m, p, a, V)$, where we have put

$$
F^{(5 k)}(q, m, p, a, V)=\sum_{v \in \mathbb{Z}(p, V)}\left(\prod_{i=1}^{p}\left[\begin{array}{c}
m(k)-a(k, i) \\
v(i)
\end{array}\right]\right) \prod_{\substack{1 \leq n \leq q \\
n \neq k}} G^{(6 n)}(q, m, p, a, v) .
$$

It follows that for any $k \in[1, q]$ we have: $F^{(6)}(q, m, p, a, V)=F^{(5 k)}(q, m, p, a, V)$. Let us summarize some of what we have proved so far.

TheOREM (3.2). Let the notation be as above. We have

$$
|\operatorname{stab}(q, m, p, a, V)|=F^{(8)}(q, m, p, a, V)=F^{(7)}(q, m, p, a, V) .
$$


Moreover, if $q$ is even then we have

$$
|\operatorname{stab}(q, m, p, a, V)|=F^{(6)}(q, m, p, a, V)=F^{(5 k)}(q, m, p, a, V)
$$

where $k$ can be any element of $[1, q]$.

Even with all these formulas, it is not clear that by giving particular values to $q, p$, and $a$, we get the desired equality between $|\operatorname{stab}(2, m, V)|$ and $\operatorname{dim}_{K} K[X]_{V}$. So we must work harder! Henceforth in this section we forget about multitableaux and restrict ourselves to the case when $q=2$. Let us fix some $k \in[1,2]$ and let $k^{\prime}=3-k$ so that $k^{\prime}$ is 2 or 1 according as $k$ is 1 or 2 . To simplify the manipulations we shall use the following notation.

$$
\begin{aligned}
& r(i)=m(k)-a(k, i) \quad \text { and } s(i)=m\left(k^{\prime}\right)-a\left(k^{\prime}, i\right), \quad \text { for } i=1,2, \ldots, p \\
& R=\sum_{i=1}^{p} r(i) \quad \text { and } S=\sum_{i=1}^{p} s(i) .
\end{aligned}
$$

With this notation, $F^{(5 k)}(q, m, p, a, V)$, in our case of $q=2$, can be written as

$$
\sum_{v \in \mathbb{Z}(p, V)}\left(\prod_{i=1}^{p}\left[\begin{array}{l}
r(i) \\
v(i)
\end{array}\right]\right) \operatorname{det}\left(\left[\begin{array}{c}
s(j) \\
v(i)+j-i
\end{array}\right]\right) \text {. }
$$

Let us denote this expression by $F^{(40)}(r, s, V)$. We shall need the following lemmas on (twisted) binomial coefficients.

Lemma (3.3). Given any integers $U$ and $A$, we have

$$
\begin{gathered}
\text { (i) } \left.\begin{array}{c}
U \\
A
\end{array}\right)=(-1)^{A}\left[\begin{array}{c}
-U-1 \\
A
\end{array}\right] \text { and }\left[\begin{array}{l}
U \\
A
\end{array}\right]=(-1)^{A}\left(\begin{array}{c}
-U-1 \\
A
\end{array}\right) . \\
\text { (ii) }\left(\begin{array}{l}
U \\
A
\end{array}\right)=\left(\begin{array}{c}
U \\
U-A
\end{array}\right) \text { iff either } U \geq 0 \text { or } U<A<0 \text {; and } \\
{\left[\begin{array}{l}
U \\
A
\end{array}\right]=\left[\begin{array}{l}
A \\
U
\end{array}\right] \text { iff either } U+A \geq 0 \text { or } U<0 \text { and } A<0 .}
\end{gathered}
$$

Proof: Obvious.

Lemma (3.4). Given any integers $U, A$, and $B$, we have

$$
\left[\begin{array}{l}
U \\
A
\end{array}\right]\left[\begin{array}{c}
U+A \\
B
\end{array}\right]=\left[\begin{array}{c}
U \\
A+B
\end{array}\right]\left[\begin{array}{l}
B \\
A
\end{array}\right]
$$

Proof: Straightforward.

LEMma (3.5). Given any positive integer $p^{\prime}$, integers $U, E$, and any $p^{\prime}$-tuple $e \in \mathbb{Z}\left(p^{\prime}, E\right)$, we have

(i) $\sum_{u \in \mathbb{Z}\left(p^{\prime}, U\right)} \prod_{i=1}^{p^{\prime}}\left(\begin{array}{l}e(i) \\ u(i)\end{array}\right)=\left(\begin{array}{l}E \\ U\end{array}\right)$

(ii) $\sum_{u \in \mathbb{Z}\left(p^{\prime}, U\right)} \prod_{i=1}^{p^{\prime}}\left[\begin{array}{l}e(i) \\ u(i)\end{array}\right]=\left[\begin{array}{c}E+p^{\prime}-1 \\ U\end{array}\right]$.

where both the sums above are essentially finite.

Proof: Let $Y$ be an indeterminate over $\mathbb{Q}$. Consider the identities

$$
\prod_{i=1}^{p^{\prime}}(1+Y)^{e(i)}=(1+Y)^{E} \text { and } \prod_{i=1}^{p^{\prime}}(1+Y)^{-e(i)-1}=(1+Y)^{-E-p^{\prime}}
$$

Use Binomial Theorem and equate the coefficients of $Y^{U}$ on both sides to deduce (i) and (ii); note that the latter follows in view of (i) of (3.3). Essential finiteness is obvious. 
Lemma (3.6). Given any integers $T, P, U, U^{\prime}$ such that $U+U^{\prime} \geq 0$ and $T-U^{\prime} \geq 0$, we have

$$
\left[\begin{array}{l}
T \\
P
\end{array}\right]\left[\begin{array}{c}
U \\
P+U^{\prime}
\end{array}\right]=\sum_{d^{*} \in \mathbb{Z}}(-1)^{d^{*}}\left[\begin{array}{c}
T+U-d^{*} \\
P
\end{array}\right]\left(\begin{array}{c}
T-U^{\prime} \\
d^{*}
\end{array}\right)\left[\begin{array}{c}
U-d^{*} \\
T
\end{array}\right]
$$

where the summation on the right is essentially finite.

Proof: Without loss of generality, we may and do assume that $P \geq 0$ and $T \geq 0$. Now by (ii) of (3.3), we see that

$$
\begin{aligned}
& \text { LHS } \\
& =\left[\begin{array}{c}
P \\
T \\
P \\
T
\end{array}\right]\left[\begin{array}{c}
P+T+U^{\prime}-T \\
U
\end{array}\right] \\
& =\sum_{d^{*} \in \mathbb{Z}}\left[\begin{array}{c}
P+T \\
U-d^{*}
\end{array}\right]\left[\begin{array}{c}
U^{\prime}-T-1 \\
d^{*}
\end{array}\right] \\
& =\sum_{d^{*} \in \mathbb{Z}}(-1)^{d^{*}}\left(\begin{array}{c}
T-U^{\prime} \\
d^{*}
\end{array}\right)\left[\begin{array}{c}
P \\
T
\end{array}\right]\left[\begin{array}{c}
P+T \\
U-d^{*}
\end{array}\right] \\
& d^{*} \in \mathbb{Z}
\end{aligned}
$$

Now, since $T-U^{\prime} \geq 0$, for the above summand to be nonzero, we must have $T-U^{\prime} \geq d^{*} \geq 0$; also $P \geq 0$ and hence $P+T-U^{\prime}-d^{*} \geq 0$. The desired equality now follows from (ii) of (3.3).

Naturally, we wish to apply the last lemma to the relevant product in $F^{(40)}(r, s, V)$. Before we do that, let us make the following elementary observations. Of these, the first observation is an easy consequence of the fact that each side of the bivector $a$ is a strictly increasing sequence, and the second one is quite obvious.

(1): For any $i, j$ in $[1, p]$, we have: $r(i)+i-j \geq 0$ and $s(j)+j-i \geq 0$.

(2): Suppose for any $i, j$ in $[1, p]$, we are given a map $A_{i j}: \mathbb{Z} \rightarrow \mathbb{Q}$ such that $\left\{d^{*} \in \mathbb{Z}: A_{i j}\left(d^{*}\right) \neq 0\right\}$ is finite. Then

$$
\prod_{j=1}^{p} \sum_{d^{*} \in \mathbb{Z}} A_{i j}\left(d^{*}\right)=\sum_{d \in \mathbb{Z}(p)} \prod_{j=1}^{p} A_{i j}(d(j))=\sum_{D \in \mathbb{Z}} \sum_{d \in \mathbb{Z}(p, D)} \prod_{j=1}^{p} A_{i j}(d(j)) .
$$

We are now ready to transform

$$
F^{(40)}(r, s, V)=\sum_{v \in \mathbb{Z}(p, V)}\left(\prod_{i=1}^{p}\left[\begin{array}{l}
r(i) \\
v(i)
\end{array}\right]\right) \operatorname{det}\left(\left[\begin{array}{c}
s(j) \\
v(i)+j-i
\end{array}\right]\right) .
$$

For a moment, let us fix some $v \in \mathbb{Z}(p, V)$, and just look at the corresponding summand. First we note that this summand can obviously be written as

$$
\operatorname{det}\left(\left[\begin{array}{l}
r(i) \\
v(i)
\end{array}\right]\left[\begin{array}{c}
s(j) \\
v(i)+j-i
\end{array}\right]\right)=\sum_{\sigma \in S_{p}} \operatorname{sgn}(\sigma) \prod_{j=1}^{p}\left[\begin{array}{l}
r(\sigma(j)) \\
v(\sigma(j))
\end{array}\right]\left[\begin{array}{c}
s(j) \\
v(\sigma(j))+j-\sigma(j)
\end{array}\right]
$$

and now for a given $\sigma \in S_{p}$, we can apply (3.6) to write the above product as

$$
\prod_{j=1}^{p} \sum_{d^{*} \in \mathbb{Z}}(-1)^{d^{*}}\left[\begin{array}{c}
r(\sigma(j))+s(j)-d^{*} \\
v(\sigma(j))
\end{array}\right]\left[\begin{array}{c}
s(j)-d^{*} \\
r(\sigma(j))
\end{array}\right]\left(\begin{array}{c}
r(\sigma(j))+\sigma(j)-j \\
d^{*}
\end{array}\right)
$$

and using observations (1) and (2) with $i=\sigma(j)$, the above expression can be written as

$$
\sum_{D \in \mathbb{Z}}(-1)^{D} \sum_{d \in \mathbb{Z}(p, D)} \prod_{j=1}^{p}\left[\begin{array}{c}
r(\sigma(j))+s(j)-d(j) \\
v(\sigma(j))
\end{array}\right]\left[\begin{array}{c}
s(j)-d(j) \\
r(\sigma(j))
\end{array}\right]\left(\begin{array}{c}
r(\sigma(j))+\sigma(j)-j \\
d(j)
\end{array}\right) .
$$


Notice that the parameter $v$ figures in only one term. So let us sum the above expression as $v$ varies over $\mathbb{Z}(p, V)$. Then, in view of (ii) of (3.5), we get

$$
\sum_{D \in \mathbb{Z}}(-1)^{D} \sum_{d \in \mathbb{Z}(p, D)}\left[\begin{array}{c}
R+S-D+p-1 \\
V
\end{array}\right]\left(\prod_{j=1}^{p}\left[\begin{array}{c}
s(j)-d(j) \\
r(\sigma(j))
\end{array}\right]\left(\begin{array}{c}
r(\sigma(j))+\sigma(j)-j \\
d(j)
\end{array}\right)\right) .
$$

Now it's time to bring back $\operatorname{sgn}(\sigma)$ and sum over $\sigma \in S_{p}$; we can move this summation sign for $\sigma$ right before the last product in the above expression, and then collate the terms to form determinants. It follows that $F^{(40)}(r, s, V)$ is equal to

$$
\sum_{D \in \mathbb{Z}}(-1)^{D} \sum_{d \in \mathbb{Z}(p, D)}\left[\begin{array}{c}
R+S-D+p-1 \\
V
\end{array}\right] \operatorname{det}\left(\left[\begin{array}{c}
s(j)-d(j) \\
r(i)
\end{array}\right]\left(\begin{array}{c}
r(i)+i-j \\
d(j)
\end{array}\right)\right) .
$$

We are pretty much done now. For any $D \in \mathbb{Z}$, let us denote the sum

$$
\sum_{d \in \mathbb{Z}(p, D)} \operatorname{det}\left(\left[\begin{array}{c}
s(i)-d(i) \\
r(j)
\end{array}\right]\left(\begin{array}{c}
r(j)+j-i \\
d(i)
\end{array}\right)\right)
$$

by $F_{D}^{(30)}(r, s)$ or alternately by $F_{D}^{(3 k)}(m, p, a)$ [recall that $r(i)=m(k)-a(k, i)$ and $\left.s(i)=m\left(k^{\prime}\right)-a\left(k^{\prime}, i\right)\right]$. Given any $d \in \mathbb{Z}(p, D)$, in order that the corresponding summand in $F_{D}^{(30)}(r, s)$ be nonzero, we must have

$$
\prod_{i=1}^{p}\left(\begin{array}{c}
r(\sigma(i))+\sigma(i)-i \\
d(i)
\end{array}\right) \neq 0 \quad \text { for some } \sigma \in S_{p}
$$

and hence, in view of the observation (1), we must have $0 \leq D \leq R$. Thus

$$
\left\{D \in \mathbb{Z}: F_{D}^{(30)}(r, s) \neq 0\right\} \subseteq[0, R] .
$$

Now we define

$$
F^{(30)}(r, s, V)=\sum_{D \in \mathbb{Z}}(-1)^{D} F_{D}^{(30)}(r, s)\left[\begin{array}{c}
V \\
R+S+p-1-D
\end{array}\right]
$$

and remark that this expression may alternately be denoted by $F^{(3 k)}(m, p, a, V)$. In view of (5) and (ii) of (3.3), we can conclude that

$$
F^{(40)}(r, s, V)=F^{(30)}(r, s, V)
$$

or in other words,

$$
F^{(5 k)}(2, m, p, a, V)=F^{(3 k)}(m, p, a, V) .
$$

Note that $F^{(3 k)}$ can be regarded as a polynomial in the parameter $V$. This is quite significant as we shall soon realize. First, we show that the much sought after equality $|\operatorname{stab}(2, m, V)|=\operatorname{dim}_{K} K[X]_{V}$ can now be deduced. To this end, let us suppose that we are in the following

Minimal Case: $p=\min \{m(1), m(2)\}$ and $a(k, i)=i=a\left(k^{\prime}, i\right)$ for all $i \in[1, p]$.

In this case, it is clear that $a \leq T$ for any $T \in \operatorname{stab}(2, m)$, and hence $\operatorname{stab}(2, m, p, a, V)$ is the same as $\operatorname{stab}(2, m, V)$. To compute $|\operatorname{stab}(2, m, V)|$ using the above formula, we may and do assume that $k \in[1,2]$ is chosen such that $p=\min \{m(1), m(2)\}=$ $m\left(k^{\prime}\right)$. For the sake of brevity, let us denote $m(k)$ by $p^{\prime}$. Given any $D \in \mathbb{Z}$ and $d \in \mathbb{Z}(p, D)$, the corresponding summand in $F_{D}^{(3 k)}(m, p, a)$ is now equal to

$$
\left\{\prod_{i=1}^{p}\left(\begin{array}{c}
p^{\prime}-i \\
d(i)
\end{array}\right)\right\} \operatorname{det}\left(\left[\begin{array}{c}
p-i-d(i) \\
p^{\prime}-j
\end{array}\right]\right) .
$$


For the above product to be nonzero, we must have $0 \leq d(i) \leq p^{\prime}-i$ for all $i \in[1, p]$; let us assume that this is the case. Now consider the $p \times p$ matrix whose $(i, j)^{\text {th }}$ entry is

$$
\left[\begin{array}{c}
p-i-d(i) \\
p^{\prime}-j
\end{array}\right]
$$

We successively make the column transformations $C_{1}-C_{2}, C_{2}-C_{3}, \ldots, C_{p-1}-C_{p}$ [where $C_{i}$ stands for the $i^{\text {th }}$ column], and use the Basic Binomial Identity (4). Then for $j \leq p-1$, the $(i, j)^{\text {th }}$ entry of the resulting matrix becomes

$$
\left[\begin{array}{c}
p-i-1-d(i) \\
p^{\prime}-j
\end{array}\right]
$$

Next, we make the column transformations $C_{1}-C_{2}, C_{2}-C_{3}, \ldots, C_{p-2}-C_{p-1}$ and use (4), following which we make the column transformations $C_{1}-C_{2}, C_{2}-$ $C_{3}, \ldots, C_{p-3}-C_{p-2}$ and use (4), and so on until we reach a stage when we just make $C_{1}-C_{2}$ and use (4). Note that the $j^{\text {th }}$ column is transformed $p-j$ times each time reducing by one the top entry in the corresponding binomial coefficient. Thus the $(i, j)^{\text {th }}$ entry in the resulting matrix equals

$$
\left[\begin{array}{c}
p-i-(p-j)-d(i) \\
p^{\prime}-j
\end{array}\right]=\left[\begin{array}{c}
j-i-d(i) \\
p^{\prime}-j
\end{array}\right]=\left(\begin{array}{c}
p^{\prime}-i-d(i) \\
p^{\prime}-j
\end{array}\right) .
$$

Since $0 \leq d(i) \leq p^{\prime}-i$, it follows that $0 \leq p^{\prime}-i-d(i)<p^{\prime}-j$ whenever $1 \leq j<i \leq p$, and hence if $j<i$, then the binomial coefficient above must be zero. Thus the resulting matrix is triangular, and therefore

$$
\operatorname{det}\left(\left[\begin{array}{c}
p-i-d(i) \\
p^{\prime}-j
\end{array}\right]\right)=\prod_{i=1}^{p}\left(\begin{array}{c}
p^{\prime}-i-d(i) \\
p^{\prime}-i
\end{array}\right) .
$$

Moreover, if $d(i)>0$ for some $i \in[1, p]$, then $0 \leq p^{\prime}-i-d(i)<p^{\prime}-i$, and hence the above product is zero; on the other hand, if $d(i)=0$ for all $i \in[1, p]$, then the above product is clearly equal to 1 . It follows that in this minimal case, for any $D \in \mathbb{Z}$, we have

and therefore

$$
F_{D}^{(3 k)}(m, p, a)= \begin{cases}1 & \text { if } D=0 \\ 0 & \text { if } D \neq 0\end{cases}
$$

$$
F^{(3 k)}(m, p, a, V)=\left[\begin{array}{c}
V \\
R+S+p-1
\end{array}\right] .
$$

Finally we note that we now have $p=m\left(k^{\prime}\right), a(k, i)=i=a\left(k^{\prime}, i\right)$, and so

$$
\begin{aligned}
R+S+p-1 & \left.=(p-1)+\sum_{i=1}^{p}[m(k)-i]+\left[m\left(k^{\prime}\right)-i\right)\right] \\
& =p-1+p m(k)+p m\left(k^{\prime}\right)-p(p+1) \\
& =m\left(k^{\prime}\right) m(k)+m\left(k^{\prime}\right)^{2}-m\left(k^{\prime}\right)^{2}-1 \\
& =m(1) m(2)-1 .
\end{aligned}
$$

Thus we have proved the following

Theorem (3.7). Given any $k \in[1,2]$ we have

(i) $|\operatorname{stab}(2, m, p, a, V)|=F^{(3 k)}(m, p, a, V)$.

(ii) $|\operatorname{stab}(2, m, V)|=\left[\begin{array}{c}V \\ m(1) m(2)-1\end{array}\right]=\operatorname{dim}_{K} K[X]_{V}$.

Remark: In Abhyankar's original proof (cf. [A1], [A3]), the above assertion (ii) is deduced from the previous assertion by first converting $F^{(3 k)}$ into another formula, called $F^{(2 k)}$, by making several transformations and using a Binomial 
Lemma similar to (3.6), and then manipulating the determinants for the above particular values of $p$ and $a$. The proof we have given here is somewhat shorter. The crucial thing, as we indicated earlier, is the fact that $F^{(3 k)}$ is a "polynomial formula" in $V$ for $|\operatorname{stab}(2, m, p, a, V)|$.

\section{Universal Determinantal Identity}

An essential ingredient of the Straightening Law, or the SBT, is the fact that any monomial in minors can be "straightened out" so as to be expressible as a sum of standard monomials in minors, i.e., monomials in minors corresponding to standard tableaux. This can be done using a certain universal determinantal identity satisfied by the minors of a matrix, which we shall now describe.

Let $m=(m(1), m(2))$ be a pair of positive integers and $X=\left(X_{i j}\right)$ be an $m(1) \times m(2)$ matrix whose entries are independent indeterminates over a field $K$, and let $K[X]$ be the corresponding ring of polynomials. If $a$ is a bivector of length $p$ and bounded by $m$, then it corresponds to a $p \times p$ minor of $X$, which we denote by $\operatorname{mor}(X, a)$; thus

$$
\operatorname{mor}(X, a)=\operatorname{det}\left(X_{a(1, i) a(2, j)}\right)_{1 \leq i, j \leq p}
$$

Given a bitableau $T=(T[1], T[2], \ldots, T[d])$ bounded by $m$, we have the corresponding monomial in minors of $X$ which we denote by $\operatorname{mom}(X, T)$; thus

$$
\operatorname{mom}(X, T)=\prod_{e=1}^{d} \operatorname{mor}(X, T[e]) .
$$

Note that $\operatorname{mom}(X, T)$ is a homogeneous polynomial in $K[X]$ and its degree is equal to the area of $T$. The so called Universal Determinantal Identity implies that for any bitableau $T$ bounded by $m$, we have

$$
\operatorname{mom}(X, T)=\sum_{S \in \operatorname{stab}(2, m)} \operatorname{fin}(m, T, S) \operatorname{mom}(X, S)
$$

where the sum is taken over all standard bitableaux $S$ bounded by $m$, and the coefficient $\operatorname{fin}(m, T, S)$ is an integer such that $\operatorname{fin}(m, T, S)=0$ for all except finitely many such $S$, and

$$
\text { fin }(m, T, S) \neq 0 \Rightarrow \text { area of } S=\text { area of } T \text {, and } S[1] \leq T[1]
$$

where the last inequality holds provided $\operatorname{dep}(T) \neq 0 \neq \operatorname{dep}(S)$. Note that the identity (6) clearly implies that

$$
\{\operatorname{mom}(X, S): S \in \operatorname{stab}(2, m)\} \text { generates } K[X]
$$

and, in view of (7), it also shows that for any nonnegative integer $V$,

$$
\{\operatorname{mom}(X, S): S \in \operatorname{stab}(2, m, V)\} \text { generates } K[X]_{V} .
$$

To see (6), we note that if a bitableau $T=(T[1], \ldots, T[d])$ of depth $d$ is not standard, then there exists $e \in[1, d-1]$ such that $T[e] \not \leq T[e+1]$. Thus it suffices to consider two bivectors $a$ and $b$ bounded by $m$ such that $a \not \leq b$, and express the product of the corresponding pair of minors as a sum of "better products" of pairs of minors. Now these bivectors may have different length, but we can reduce to the case of maximal size minors by means of the following lemma whose proof is left as an exercise. Let us first assume, without loss of generality, that $m(1) \leq m(2)$.

Lemma (4.1). Given any bivector a bounded by $m$ and of length $p$, let $\alpha_{1}, \alpha_{2}, \ldots$, $\alpha_{m(1)-p}$ be integers such that $\alpha_{1}<\alpha_{2}<\cdots<\alpha_{m(1)-p}$ and

$$
[1, m(1)] \backslash\{a(1,1), \ldots, a(1, p)\}=\left\{\alpha_{1}, \ldots, \alpha_{m(1)-p}\right\} .
$$


Let $\beta_{1}, \beta_{2}, \ldots, \beta_{m(1)}$ be defined by

$$
\beta_{j}= \begin{cases}a(2, j) & \text { if } 1 \leq j \leq p \\ m(1)+m(2)+1-\alpha_{m(1)+1-j} & \text { if } p+1 \leq j \leq m(1) .\end{cases}
$$

Then $\beta=\left(\beta_{1}, \beta_{2}, \ldots, \beta_{m(1)}\right)$ is a univector bounded by $m(1)+m(2)$ and of length $m(1)$. Moreover, the above correspondence $a \mapsto \beta$ is an order-preserving bijection of the set of all bivectors bounded by $m$ onto the set of all univectors bounded by $m(1)+m(2)$ and of length $m(1)$. Furthermore, if we let $\bar{X}=\left(X \mid I^{*}\right)$ be the $m(1) \times$ $(m(1)+m(2))$ matrix obtained by adjoining the $m(1) \times m(1)$ antiidentity matrix $I^{*}=\left(\delta_{i m(1)+1-j}\right)$ [where $\delta$ is the usual Kronecker delta] to $X$, then $\operatorname{mor}(X, a)=$ $\pm \operatorname{det}[\beta]$, where $[\beta]$ denotes the $m(1) \times m(1)$ submatrix of $\bar{X}$ formed by the columns whose indices are $\beta_{1}, \ldots, \beta_{m(1)}$.

To obtain the desired identity for $m(1) \times m(1)$ minors of $\bar{X}$ [or for that matter, any $m(1) \times(m(1)+m(2))$ matrix], we first note that univectors bounded by $m(1)+m(2)$ and of length $m(1)$ can alternatively be thought of as subsets of $[1, m(1)+m(2)]$ of cardinality $m(1)$, and we may tacitly do so when it is convenient. Given a subset $T^{*}$ of $[1, m(1)+m(2)]$ of cardinality $m(1)$, or in other words, a univector $T^{*}$ bounded by $m(1)+m(2)$ and of length $m(1)$, we let $\left[T^{*}\right]$ denote the $m(1) \times m(1)$ submatrix of $\bar{X}$ corresponding to $T^{*}$. Now let $T_{1}^{*}$ and $T_{2}^{*}$ be univectors bounded by $m(1)+m(2)$ and of length $m(1)$ such that $T_{1}^{*} \not \leq T_{2}^{*}$. Then there exists a unique $v \in[1, m(1)]$ such that

$$
T_{1}^{*}(1, i) \leq T_{2}^{*}(1, i) \text { for } 1 \leq i<v \text { and } T_{1}^{*}(1, v)>T_{2}^{*}(1, v) .
$$

Let us take

$$
B_{1}^{*}=\left\{T_{1}^{*}(1, i): v \leq i \leq m(1)\right\} \text { and } B_{2}^{*}=\left\{T_{2}^{*}(1, i): 1 \leq i \leq v\right\}
$$

and consider the $2 m(1) \times 2 m(1)$ matrix

$$
y=\begin{array}{|c|c|}
\hline\left[T_{1}^{*}\right] & {\left[\widehat{B}_{2}^{*}\right]} \\
\hline\left[\widehat{B}_{1}^{*}\right] & {\left[T_{2}^{*}\right]} \\
\hline
\end{array}
$$

where $\left[\widehat{B}_{1}^{*}\right]\left(\right.$ resp: $\left.\left[\widehat{B}_{2}^{*}\right]\right)$ denotes the $m(1) \times m(1)$ submatrix of $\left[T_{1}^{*}\right]\left(\right.$ resp: $\left.\left[T_{2}^{*}\right]\right)$ obtained by taking the columns corresponding to $B_{1}^{*}$ (resp: $B_{2}^{*}$ ) and filling the remaining columns by zeros. Thus the first $v$ columns of $\left[\widehat{B}_{2}^{*}\right]$ are the same as those of $\left[T_{2}^{*}\right]$, whereas the last $m(1)-v+1$ columns of $\left[\widehat{B}_{1}^{*}\right]$ are the same as those of $\left[T_{1}^{*}\right]$. Now we can expand $y$ by Laplace development considering the two blocks formed by the first $m(1)$ rows and by the last $m(1)$ rows. This gives

$$
\operatorname{det} y=\sum_{\substack{A_{*}^{*} \subseteq B_{k}^{*} \\\left|A_{1}^{*}\right|=\left|A_{2}^{*}\right|}} \pm \operatorname{det}\left(\left[\left(T_{1}^{*} \backslash A_{1}^{*}\right) \cup A_{2}^{*}\right]\right) \operatorname{det}\left(\left[\left(T_{2}^{*} \backslash A_{2}^{*}\right) \cup A_{1}^{*}\right)\right.
$$

where the sum is taken over all pairs $A^{*}=\left(A_{1}^{*}, A_{2}^{*}\right)$ with $A_{1}^{*} \subseteq B_{1}^{*}, A_{2}^{*} \subseteq B_{2}^{*}$, and $\left|A_{1}^{*}\right|=\left|A_{2}^{*}\right|$. On the other hand, if in $y$ we subtract the lower block from the upper block [i.e., $(m(1)+i)^{\text {th }}$ row from $i^{\text {th }}$ row, for $1 \leq i \leq m(1)$ ], and expand the resulting matrix by Laplace expansion as before, then in view of the fact that

$$
\left|B_{1}^{*}\right|+\left|B_{2}^{*}\right|>m(1)
$$

we see that in each term one of the submatrices has a column of zero, and hence

$$
\operatorname{det} y=0 \text {. }
$$


Combining this with (11), and pulling out the term corresponding to $A_{1}^{*}=\emptyset=A_{2}^{*}$, we get

$$
\operatorname{det}\left(\left[T_{1}^{*}\right]\right) \operatorname{det}\left(\left[T_{2}^{*}\right]\right)=\sum_{\substack{A_{k}^{*} \subseteq B_{*}^{*} \\\left|A_{1}^{*}\right|=\left|A_{2}^{*}\right| \neq 0}} \pm \operatorname{det}\left(\left[S_{1}^{*}\left(T_{1}^{*}, A_{1}^{*}\right)\right]\right) \operatorname{det}\left(\left[S_{2}^{*}\left(T_{2}^{*}, A_{2}^{*}\right)\right]\right)
$$

where the sum is taken over all nonempty subsets $A_{1}^{*}, A_{2}^{*}$ of $B_{1}^{*}, B_{2}^{*}$ respectively such that $\left|A_{1}^{*}\right|=\left|A_{2}^{*}\right|$, and for any such pair $\left(A_{1}^{*}, A_{2}^{*}\right)$, we have let $S_{1}^{*}\left(T_{1}^{*}, A_{1}^{*}\right)$ be the univector corresponding to $\left(T_{1}^{*} \backslash A_{1}^{*}\right) \cup A_{2}^{*}$ and $S_{2}^{*}\left(T_{2}^{*}, A_{2}^{*}\right)$ be the univector corresponding to $\left(T_{2}^{*} \backslash A_{2}^{*}\right) \cup A_{1}^{*}$. By the choice of $B_{1}^{*}$ and $B_{2}^{*}$, and the strict inequality in (10), it follows that for any $\left(A_{1}^{*}, A_{2}^{*}\right)$ as above, we have

$$
S_{1}^{*}\left(T_{1}^{*}, A_{1}^{*}\right)<T_{1}^{*} .
$$

Thus, in view of (15), we can deduce (6) and (7) by repeated applications of (14); this is facilitated by restricting to bitableaux of a fixed area $V$ which enables us to work only in the finite set $\operatorname{stab}(2, m, V)$. Consequently, we get (8) and (9). In [A3], the condition (13) is generalized so as to obtain the identity (14) with weaker assumptions; an identity like (14), which clearly holds for any matrix of appropriate size, may be referred to as a universal determinantal identity. For details concerning this section, see [A3]. As remarked earlier, the ideas outlined here are fairly classical and go back at least to Hodge [Ho]; for proofs of (8) along similar lines, see [DEP], [DKR], [Se].

Finally, we use the results of the previous section to prove the

Standard Basis Theorem (4.2). Given any nonnegative integer $V,\{\operatorname{mom}(X, S)$ : $S \in \operatorname{stab}(2, m, V)\}$ forms a $K$-vector space basis of $K[X]_{V}$. Moreover, $\{\operatorname{mom}(X, S)$ : $S \in \operatorname{stab}(2, m)\}$ forms a $K$-vector space basis of $K[X]$.

Proof: The first assertion follows from (8) and (ii) of (3.7), and the second assertion follows from the first one.

For another proof, also based on enumeration, of the above theorem, see [AG].

\section{IndeXed Monomials}

In this section, we describe a certain set of monomials in $X=\left(X_{i j}\right)_{\substack{1 \leq i \leq m(1) \\ 1 \leq j \leq m(2)}}$ and indicate how this set can be enumerated. It turns out that this set of monomials has the same cardinality as that of $\operatorname{stab}(2, m, p, a, V)$. Here, as usual, $m=(m(1), m(2))$ is a pair of positive integers, $p$ is a positive integer, $a$ is a bivector bounded by $m$ and of length $p$, and $V$ is a nonnegative integer. We proceed to give a brief sketch of the main ideas of Abhyankar concerning the enumeration of these so called indexed monomials. For details, see Sections 5 and 8 of [A3]. Some applications of the results in this section, which may be regarded as a motivation to obtain these results, are indicated in the next section.

Let us begin by noting that a monomial in the $m(1) m(2)$ variables $X_{i j}$ 's can be thought of as a map from the rectangle $[1, m(1)] \times[1, m(2)]$ into the set $\mathbb{N}$ of nonnegative integers. Indeed, if $t:[1, m(1)] \times[1, m(2)] \rightarrow \mathbb{N}$ is any map, then

$$
X^{t} \stackrel{\text { def }}{=} \prod_{y} X_{y(1) y(2)}^{t(y)},
$$

where the product is taken over all pairs $y=(y(1), y(2)) \in[1, m(1)] \times[1, m(2)]$, is a monomial in the $X_{i j}$ 's. Conversely, a monomial in $X_{i j}$ 's obviously corresponds to such a map. In this terminology, we have the notions of absolute value abs $(t)$ and support $\operatorname{supp}(t)$ of a monomial $t$, which are defined as follows.

$$
\operatorname{abs}(t)=\operatorname{deg}\left(X^{t}\right)=\sum_{y \in[1, m(1)] \times[1, m(2)]} t(y)
$$


and

$$
\operatorname{supp}(t)=\{y \in[1, m(1)] \times[1, m(2)]: t(y) \neq 0\} .
$$

Let $\operatorname{mon}(2, m)$ denote the set of all maps $t:[1, m(1)] \times[1, m(2)] \rightarrow \mathbb{N}$, and given any nonnegative integer $V$, let $\operatorname{mon}(2, m, V)$ be the set of all elements $t \in \operatorname{mon}(2, m)$ such that $\operatorname{abs}(t)=V$. Now we have the key notion of index of a subset of $[1, m(1)] \times$ $[1, m(2)]$; the index of a monomial will be the index of its support, and roughly speaking, it is equal to the largest integer $j$ such that the principal diagonal of a $j \times j$ minor of $X$ divides this monomial. More precisely, for any subset $Y$ of $[1, m(1)] \times[1, m(2)]$, we define

$$
\begin{aligned}
\operatorname{ind}(Y)=\max \{j: & \exists y_{1}, \ldots, y_{j} \in Y \text { such that } y_{i}(k)<y_{i+1}(k) \\
& \text { for all } k \in[1,2] \text { and } i \in[1, j-1]\}
\end{aligned}
$$

and for any monomial $t \in \operatorname{mon}(2, m)$, we define

$$
\operatorname{ind}(t)=\operatorname{ind}(\operatorname{supp}(t)) \text {. }
$$

Given a bivector $a$ bounded by $m$ and of length $p$, we can draw vertical and horizontal lines in the rectangle $[1, m(1)] \times[1, m(2)]$ emanating from $a(k, i)$, where $k$ can be 1 or 2 , and $i$ varies between 1 and $p$, and consider the monomials satisfying an index condition when restricted to the region above [or to the left of] $a(k, i)$. More precisely, given any $k \in[1,2], i \in[1, p]$, and $t \in \operatorname{mon}(2, m)$, we let

$$
t_{k i}=\{y \in \operatorname{supp}(t): y(k)<a(k, i)\}
$$

and we define

$$
\begin{aligned}
\operatorname{mon}(2, m, p, a)=\{t \in \operatorname{mon}(2, m) \quad: & \operatorname{ind}(t) \leq p \text { and } \operatorname{ind}\left(t_{k i}\right)<i \\
& \text { for all } k \in[1,2], \text { and } i \in[1, p]\}
\end{aligned}
$$

and for any $V \in \mathbb{N}$, we define

$$
\operatorname{mon}(2, m, p, a, V)=\operatorname{mon}(2, m, p, a) \cap \operatorname{mon}(2, m, V) .
$$

The elements of this set may be referred to as indexed monomials. We wish to find the cardinality of this set. The basic strategy is similar to that employed in finding $|\operatorname{stab}(q, m, p, a, V)|$, namely, to first find recurrence relations satisfied by the cardinality, and then discover an explicit formula satisfying the same recurrence relations. To obtain a recurrence relation satisfied by $|\operatorname{mon}(2, m, p, a, V)|$, we enlarge the rectangle $[1, m(1)] \times[1, m(2)]$ on one of its sides, say the $k^{\text {th }}$ side, and look at $\left[1, m^{*}(1)\right] \times\left[1, m^{*}(2)\right]$ where $m^{*}(k)=m(k)+1$ and $m^{*}\left(k^{\prime}\right)=m\left(k^{\prime}\right)\left[k^{\prime}\right.$ equals 2 or 1 according as $k$ is 1 or 2 , i.e., $k^{\prime}=3-k$. Now a monomial on the larger rectangle can be split into a monomial on the original rectangle and a monomial defined on the remaining line. The latter can be counted easily. Keeping track of conditions on index and such, it is not hard to see that we get the recurrence relations described below.

Basically, we have 2 recurrence relations, of which the first one tells how to express $\left|\operatorname{mon}\left(2, m^{*}, p, a^{*}, V\right)\right|$ in terms of $|\operatorname{mon}(2, m, p, a, V)|$ when the vector $a^{*}$ has length $p$ and is such that $\left[m^{*}(k)-a^{*}(k, p)\right]=[m(k)-a(k, p)]+1$, and the second one tells us how to express $\left|\operatorname{mon}\left(2, m^{*}, p+1, a^{*}, V\right)\right|$ in terms of $|\operatorname{mon}(2, m, p, a, V)|$ when the vector $a^{*}$ has length $p+1$ and is such that $\left[m^{*}(k)-a^{*}(k, p+1)\right]=0$. To describe both the relations in one stroke, we can consider a bivector $b$ of length $p+1$, and, for any subset $u$ of $[1, p]$, let

$$
\begin{aligned}
M[p, b, k, u]=\{a: \quad & a(k, i)=b(k, i) \forall i \in[1, p] \text { and } \\
& b\left(k^{\prime}, j\right)=a\left(k^{\prime}, j\right) \forall j \in[1, p] \backslash u, \text { and } \\
& \left.b\left(k^{\prime}, i\right)<a\left(k^{\prime}, i\right)<b\left(k^{\prime}, i+1\right) \forall i \in u\right\} ;
\end{aligned}
$$


note that the elements of $M[p, b, k, u]$ are bivectors of length $p$. For any $U \in[0, p]$, let

$$
M(p, b, k, U)=\bigcup_{\substack{u \subseteq[1, p] \\|u|=U}} M[p, b, k, u] .
$$

Recall that $m^{*}=\left(m^{*}(1), m^{*}(2)\right)$ is given by $m^{*}(k)=m(k)+1$ and $m^{*}\left(k^{\prime}\right)=m\left(k^{\prime}\right)$, and let a positive integer $p^{*}$ and a bivector $a^{*}$ bounded by $m^{*}$ and of length $p^{*}$ be given. Consider the following conditions on $p^{*}$ and $b$.

$$
\left\{\begin{array}{l}
p^{*}=p, a^{*}\left(k, p^{*}\right) \neq m^{*}(k), b(\hat{k}, i)=a^{*}(\hat{k}, i), \text { and } \\
b(\hat{k}, p+1)=m(\hat{k})+1 \text { for all } \hat{k} \in[1,2] \text { and } i \in[1, p]
\end{array}\right.
$$

Or

$$
p^{*}=p+1, a^{*}\left(k, p^{*}\right)=m^{*}(k), \text { and } b=a^{*} .
$$

Finally, let

$$
C^{*}=p+m\left(k^{\prime}\right)-b\left(k^{\prime}, p+1\right)+\sum_{i=1}^{p}\left[a\left(k^{\prime}, i\right)-b\left(k^{\prime}, i\right)\right] .
$$

Now the said recurrence relations can be stated as follows.

Lemma (5.1). Let the notation be as above. Assume that either (16) holds or (17) holds. Then

$$
\left|\operatorname{mon}\left(2, m^{*}, p^{*}, a^{*}, V\right)\right|=\sum_{U=0}^{p} \sum_{a \in M(p, b, k, U)} \sum_{\substack{v_{1}, v_{2} \in \mathbb{N} \\
v_{1}+v_{2}=V}}\left|\operatorname{mon}\left(2, m, p, a, v_{1}\right)\right|\left[\begin{array}{c}
C^{*} \\
v_{2}-U
\end{array}\right] .
$$

Let us also note that we can easily compute $|\operatorname{mon}(2, m, p, a, V)|$ in the initial case, i.e.,

$$
\left\{\begin{array}{l}
\text { if the bivector } a \text { has length } p=1, \text { and if } \\
m(k)-a(k, p)=0, \text { then for every } V \in \mathbb{N}, \\
|\operatorname{mon}(2, m, p, a, V)|=\left[\begin{array}{c}
m\left(k^{\prime}\right)-a\left(k^{\prime}, 1\right) \\
V
\end{array}\right] .
\end{array}\right.
$$

By making double induction on $p$ and $m(k)-a(k, p)$, we see that the cardinality of $\operatorname{mon}(2, m, p, a, V)$ is determined by the above recurrence relations and initial condition. In 1984, Abhyankar obtained a formula satisfying the same properties; this formula is denoted by $F^{(1 k)}(m, p, a, V)$ and it can be defined as follows.

$$
F^{(1 k)}(m, p, a, V)=\sum_{D \in \mathbb{Z}}(-1)^{D} F_{D}^{(1 k)}(m, p, a)\left[\begin{array}{c}
V \\
C(m, p, a)-D
\end{array}\right]
$$

where $C(m, p, a)=(p-1)+C_{1}+C_{2}$ with $C_{k}=\sum_{i=1}^{p}[m(k)-a(k, i)]$, and for any $D \in \mathbb{Z}$

$$
F_{D}^{(1 k)}(m, p, a)=\sum_{E \in \mathbb{Z}}(-1)^{C_{k}-E}\left(\begin{array}{c}
E \\
D+E-C_{k}
\end{array}\right) H_{E}^{(1 k)}
$$

where for any $E \in \mathbb{Z}$,

$$
H_{E}^{(1 k)}=\sum_{e \in \mathbb{Z}(p, E)} \operatorname{det}\left(\left[\begin{array}{c}
m(k)-a(k, j)+j-i \\
m(k)-a(k, j)-e(i)
\end{array}\right]\right) .
$$

It may be noted that all the summations above are essentially finite. Thus we have the following

Theorem (5.2). For any $k \in[1,2]$, we have $|\operatorname{mon}(2, m, p, a, V)|=F^{(1 k)}(m, p, a, V)$. 
Now we already have enumerative formulas for $|\operatorname{stab}(2, m, p, a, V)|$, and notable among those is $F^{(3 k)}(m, p, a, V)$ which is a polynomial in $V$ and which has an expression similar to $F^{(1 k)}(m, p, a, V)$. Transforming the summations and using some properties of binomial coefficients, in a manner somewhat similar to that in Section 3, Abhyankar proved that for every $D \in \mathbb{Z}$, we have

$$
F_{D}^{(3 k)}(m, p, a)=F_{D}^{(1 k)}(m, p, a) .
$$

Combining this with (3.7) and (5.2), we get the following

TheOrem (5.3). The sets $\operatorname{stab}(2, m, p, a, V)$ and $\operatorname{mon}(2, m, p, a, V)$ have the same cardinality. Moreover, the common cardinality is given by the equivalent formulas $F^{(3 k)}(m, p, a, V)$ or $F^{(1 k)}(m, p, a, V)$.

Note that from (5.3), we can easily deduce that $|\operatorname{stab}(2, m, V)|=\operatorname{dim}_{K} K[X]_{V}$; indeed, $\operatorname{mon}(2, m, V)$ simply consists of all monomials of degree $V$ in $m(1) m(2)$ indeterminates.

\section{Determinantal Ideals and their Hilbert Functions}

Let $K$ be a field and let $X=\left(X_{i j}\right)$ be an $m(1) \times m(2)$ matrix whose entries are independent indeterminates over $K$, and let $K[X]$ denote the corresponding ring of polynomials. Given a positive integer $p \leq \min \{m(1), m(2)\}$, let $I_{p}$ denote the ideal in $K[X]$ generated by all $p \times p$ minors of $X$. Also, given any bivector $a$ bounded by the pair $m=(m(1), m(2))$ of positive integers and of length $p$, let $I(p, a)$ denote the ideal in $K[X]$ generated by all $(p+1) \times(p+1)$ minors of $X$, all $i \times i$ minors of the submatrix $\left(X_{u v}\right)_{\substack{1 \leq u \leq a(1, i)-1 \\ 1 \leq v \leq m(2)}}$, and all $j \times j$ minors of $\left(X_{u v}\right)_{\substack{1 \leq u \leq m(1) \\ 1 \leq v \leq a(2, j)-1}}$, where $i$ and

$j$ range over $[1, p]$. Equivalently, $I(p, a)$ is the ideal in $K[X]$ generated by $\operatorname{mor}(X, b)$ with $b$ ranging over the set of all bivectors bounded by $m$ such that $a \not z b$ (verify!). Note that in the case when $a(1, i)=i=a(2, i)$ for all $i \in[1, p]$, we have $I(p, a)=$ $I_{p+1}$. The study of the ideals $I_{p}$ is classical, and the primality of such ideals was first proved by E. Pascal in 1888 [P] in the form of Second Fundamental Theorem of Invariant Theory. For more recent proofs, see: [M], [HE], [DEP], [A1]. It may be noted that ideals similar to $I(p, a)$ have also been studied in [HE], [Mus2]. In this section, we shall describe explicit $K$-vector space bases for the ideal $I(p, a)$ and its residue class ring $K[X] / I(p, a)$, using the ideas of straightening. $I(p, a)$ is clearly a homogeneous ideal, and these bases induce bases for the homogeneous components of $I(p, a)$ and $K[X] / I(p, a)$. In fact, the set $\{\operatorname{mom}(X, S): S \in \operatorname{stab}(2, m, p, a, V)\}$ is a $K$-basis for $K[X]_{V} / I(p, a)_{V}$. Consequently, $F^{(3 k)}(m, p, a, V)$ gives the Hilbert function of $I(p, a)$. Thus the ideal $I(p, a)$ is Hilbertian in the sense that its Hilbert function equals its Hilbert polynomial for all values of the parameter $V$. As another consequence of the Straightening Law, we can deduce that $I(p, a)$ is, in fact, a homogeneous prime ideal and the corresponding projective variety is rational, i.e., the quotient field of $K[X] / I(p, a)$ is a pure transcendental extension of $K$. The indexed monomials can be used to give another basis for $I(p, a)$. This basis has the advantage that it can be adapted to give bases for a more general class of ideals such as the ladder determinantal ideal $I_{p}(L)$ discussed in the Introduction; moreover, we can also use it to prove the primality of these ideals.

To describe a basis of $K[X] / I(p, a)$, let us define

$$
\operatorname{stab}(2, m, p, a)=\{T \in \operatorname{stab}(2, m): a \leq T[e] \text { for all } e \in[1, \operatorname{dep}(T)]\} .
$$

TheOREM (6.1). Given $m, p, a$ as above, we have the following.

(i) $\{\operatorname{mom}(X, S): S \in \operatorname{stab}(2, m) \backslash \operatorname{stab}(2, m, p, a)\}$ is a $K$-basis of $I(p, a)$, whereas $\{\operatorname{mom}(X, S): S \in \operatorname{stab}(2, m, p, a)\}$ is a $K$-basis of $K[X] / I(p, a)$. 
(ii) For every $V \in \mathbb{N},\{\operatorname{mom}(X, S): S \in \operatorname{stab}(2, m, V) \backslash \operatorname{stab}(2, m, p, a, V)\}$ is a $K$-basis of $I(p, a)_{V}$, whereas $\{\operatorname{mom}(X, S): S \in \operatorname{stab}(2, m, p, a, V)\}$ is a $K$-basis of $K[X]_{V} / I(p, a)_{V}$.

(iii) For every $V \in \mathbb{N}, \operatorname{dim}_{K} K[X]_{V} / I(p, a)_{V}=|\operatorname{stab}(2, m, p, a, V)|$.

Proof: Since $I(p, a)$ is generated by $\{\operatorname{mor}(X, b): a \not \leq b\}$, given any polynomial $B \in I(p, a)$, we can find bivectors $b_{1}, \ldots, b_{n}$ with $a \not \leq b_{i}$ for each $i \in[1, n]$, elements $r_{1}, \ldots, r_{n}$ in $K$, and (usual) monomials $M_{1}, \ldots, M_{n}$ in the $X_{i j}$ 's such that

$$
B=\sum_{i=1}^{n} r_{i} \operatorname{mor}\left(X, b_{i}\right) M_{i}
$$

For each $i \in[1, n]$, the monomial $M_{i}$ can be regarded as a monomial in minors of $X$ corresponding to a bitableau $U_{i}$ such that every row of $U_{i}$ is of length 1 . Now if we let $T_{i}$ denote the bitableau obtained by augmenting $U_{i}$ with $b_{i}$, i.e, $T_{i}[1]=b_{i}$ and $T_{i}[e]=U_{i}[e-1]$ for $e=2,3, \ldots, \operatorname{dep}\left(T_{i}\right)=\operatorname{dep}\left(U_{i}\right)+1$, then we have $\operatorname{mor}\left(X, b_{i}\right) M_{i}=\operatorname{mom}\left(X, T_{i}\right)$. By (6) and (7) of Section 4, we see that each $\operatorname{mom}\left(X, T_{i}\right)$ can be written as an integral linear combination of $\operatorname{mom}\left(X, S_{i j}\right)$ for some $S_{i j} \in \operatorname{stab}(2, m)$ with $S_{i j}[1] \leq T_{i}[1]=b_{i}$. Hence we can find $S_{1}, \ldots, S_{N}$ in $\operatorname{stab}(2, m)$ and elements $t_{1}, \ldots, t_{N}$ in $K$ such that $a \not \leq S_{i}[1]$ (so that $S_{i} \in$ $\operatorname{stab}(2, m) \backslash \operatorname{stab}(2, m, p, a))$, and write

$$
B=\sum_{i=1}^{N} t_{i} \operatorname{mom}\left(X, S_{i}\right)
$$

Moreover, given any $S \in \operatorname{stab}(2, m) \backslash \operatorname{stab}(2, m, p, a)$, we have $\operatorname{dep}(S) \neq 0$ and $a \not \leq S[1]$. Hence one of the following statements must hold.

1. $\exists i \in[1, p]$ such that $S[1](1, \ell) \geq a(1, \ell)$ for $1 \leq \ell<i$, and $S[1](1, i)<a(1, i)$.

2. $\exists j \in[1, p]$ such that $S[1](2, \ell) \geq a(2, \ell)$ for $1 \leq \ell<j$, and $S[1](2, j)<a(2, j)$.

3 . the length of $S[1]$ is $\geq p+1$.

In each of these cases, it is easily seen that $\operatorname{mor}(X, S[1])$ can be written as a sum of multiples of the minors which are amongst generators of $I(p, a)$; therefore $\operatorname{mor}(X, S[1]) \in I(p, a)$. Thus $\{\operatorname{mom}(X, S): S \in \operatorname{stab}(2, m) \backslash \operatorname{stab}(2, m, p, a)\}$ is a subset of $I(p, a)$. Note that this set is linearly independent being a subset of the basis $\{\operatorname{mom}(X, S): S \in \operatorname{stab}(2, m)\}$ of $K[X]$. This proves (i). Assertions (ii) and (iii) follow immediately from (i).

Corollary (6.2). For any $k \in[1,2], F^{(3 k)}(m, p, a, V)$ is the Hilbert function as well as the Hilbert polynomial of $I(p, a)$. In particular, if $\mathcal{V}(p, a)$ denotes the projective variety corresponding to $I(p, a)$, then

$$
\operatorname{dim} \mathcal{V}(p, a)=\operatorname{deg}_{V} F^{(3 k)}(m, p, a, V)=(p-1)+\sum_{\hat{k}=1}^{2} \sum_{i=1}^{p}[m(\hat{k})-a(\hat{k}, i)]
$$

and the degree of $\mathcal{V}(p, a)$ equals $F_{0}^{(3 k)}(m, p, a)$.

Proof: Follows from (6.1) and a well-known theorem of Hilbert (see, for example, pp. 235-236 of $[\mathrm{ZS}])$.

Now we discuss the primality of the ideal $I(p, a)$. A key step in Abhyankar's proof is the following

Lemma (6.3). The $p \times p$ minor of $X$ corresponding to $a$ is not a zero divisor in $K[X] / I(p, a)$, i.e.,

$$
B \in K[X], \operatorname{mor}(X, a) B \in I(p, a) \Rightarrow B \in I(p, a) .
$$


Proof: By SBT, we can write $B=B_{0}+B_{1}$ with

$$
B_{0}=\sum_{i=1}^{n_{0}} r_{i} \operatorname{mom}\left(X, T_{i}\right) \quad \text { and } \quad B_{1}=\sum_{j=1}^{n_{1}} t_{j} \operatorname{mom}\left(X, S_{j}\right)
$$

where $T_{i} \in \operatorname{stab}(2, m) \backslash \operatorname{stab}(2, m, p, a), S_{j} \in \operatorname{stab}(2, m, p, a), r_{i}, s_{j}$ are elements of $K$ for $1 \leq i \leq n_{0}, 1 \leq j \leq n_{1}$. Assume that $\operatorname{mor}(X, a) B \in I(p, a)$. By (6.1), we see that $B_{0} \in I(p, a)$, and hence $\operatorname{mor}(X, a) B_{1} \in I(p, a)$. But we clearly have

$$
\operatorname{mor}(X, a) B_{1}=\sum_{j=1}^{n_{1}} t_{j} \operatorname{mom}\left(X, S_{j}^{*}\right)
$$

where for each $j \in\left[1, n_{1}\right], S_{j}^{*}$ is the bitableau obtained by augmenting $a$ with $S_{j}$. Since $a \leq S_{j}, S_{j}^{*} \in \operatorname{stab}(2, m, p, a)$. In view of (6.1), it follows that we must have $t_{j}=0$ for each $j \in\left[1, n_{1}\right]$. Thus $B_{1}=0$, and hence $B=B_{0} \in I(p, a)$.

Once we have this lemma at our disposal, proving the primality is basically a clever application of Elementary Linear Algebra. For the sake of simplicity, we just consider the ideal $I_{p+1}$ and outline a proof that it is prime. Proof in the general case, i.e., for $I(p, a)$, is based on analogous ideas and later we shall indicate how one proceeds in this case. Just as before, [A3] is the reference for greater details. Now we introduce some obvious notation.

Notation. Given any subset $Y$ of $[1, m(1)] \times[1, m(2)]$, by $K[Y]$ we denote the ring of polynomials in the indeterminates $X_{i j}$ with $(i, j)$ ranging over $Y$, and by $K(Y)$ we denote the quotient field of $K[Y]$. By $E[p]$ we will denote the bivector of length $p$ given by $E[p](1, i)=i=E[p](2, i)$ for all $i \in[1, p]$. Note that $I(p, E[p])=$ $I_{p+1}$.

To show that $I_{p+1}$ is prime, we define a subset $y[p+1]$ of $[1, m(1)] \times[1, m(2)]$, and a homomorphism $f[p+1]: K[X] \rightarrow K(y[p+1])$, and prove that $I_{p+1}$ equals the kernel of $f[p+1]$. The set $y[p+1]$ is obtained by cutting out the subrectangle $[p+1, m(1)] \times[p+1, m(2)]$, i.e.,

$$
y[p+1]=\{(i, j) \in[1, m(1)] \times[1, m(2)]: \text { either } i \leq p \text { or } j \leq p\} .
$$

If $(i, j) \notin y[p+1]$, then we consider the $(p+1) \times(p+1)$ submatrix $M_{i j}$ of $X$ formed by the rows $1,2, \ldots, p, i$ and the columns $1,2, \ldots, p, j$. Clearly, we can find a unique element $\widetilde{A}[X, p+1, i, j] \in K[y[p+1]]$ such that

$$
\operatorname{det} M_{i j}=\operatorname{mor}(X, E[p]) X_{i j}-\widetilde{A}[X, p+1, i, j] \text {. }
$$

We define $f[p+1]$ to be the unique $K$-homomorphism of $K[X]$ into $K(y[p+1])$ such that

$$
f[p+1]\left(X_{i j}\right)= \begin{cases}X_{i j} & \text { if }(i, j) \in y[p+1] \\ \frac{\widetilde{A}[X, p+1, i, j]}{\operatorname{mor}(X, E[p])} & \text { if }(i, j) \notin y[p+1] .\end{cases}
$$

Let us write $x_{i j}=f[p+1]\left(X_{i j}\right)$. To show that $I_{p+1} \subseteq \operatorname{ker} f[p+1]$, it suffices to show that the rank of the $m(1) \times m(2)$ matrix $x=\left(x_{i j}\right)$ is equal to $p$. Given any $i \in[p+1, m(1)]$, consider the $(p+1) \times m(2)$ submatrix, say $\widehat{M}$, formed by the rows $1,2, \ldots, p, i$ of $x$. Since $\operatorname{mor}(x, E[p])=\operatorname{mor}(X, E[p]) \neq 0$, the first $p$ rows of $x$ are linearly independent, and so $\operatorname{rank}(\widehat{M}) \geq p$. On the other hand, for any $j \in[p+1, m(2)]$, the determinant of the $(p+1) \times(p+1)$ submatrix of $\widehat{M}$ formed by its columns $1,2, \ldots, p, j$, is zero, and hence the $j^{\text {th }}$ column of $\widehat{M}$ is linearly dependent on the first $p$ columns of $\widehat{M}$, and therefore $\operatorname{rank}(\widehat{M}) \leq p$. This shows that the $i^{\text {th }}$ row of $x$ is linearly dependent on the first $p$ rows of $x$, for any $i \in[p+1, m(1)]$. It follows that $\operatorname{rank}(x)=p$ and $I_{p+1} \subseteq \operatorname{ker} f[p+1]$. To prove the 
reverse inclusion, let us take any polynomial $B(X)$ in $K[X]$. In view of (19), we see that upon multiplying $B(X)$ by a large enough power of $\operatorname{mor}(X, E[p])$, we have

$$
\operatorname{mor}(X, E[p])^{e} B(X)=B^{* *}(X)+\sum_{(i, j) \notin y[p+1]} B_{i j}^{*}(X) \operatorname{det} M_{i j}
$$

for some $B^{* *}(X) \in K[y[p+1]]$, polynomials $B_{i j}^{*}(X) \in K[X]$, and a nonnegative integer $e$. Suppose $B(X) \in \operatorname{ker} f[p+1]$. Since det $M_{i j} \in I_{p+1}$ and $I_{p+1} \subseteq \operatorname{ker} f[p+1]$, it follows that $B^{* *}(X) \in \operatorname{ker} f[p+1]$. But $f[p+1]$ is identity on $K[y[p+1]]$, so we must have $B^{* *}(X)=0$. Hence $\operatorname{mor}(X, E[p])^{e} B(X) \in I_{p+1}$, and so, in view of (6.3), we conclude that $B(X) \in I_{p+1}$. This proves that $I_{p+1}$ is prime.

In the general case, the ideal $I(p, a)$ is proved to be the kernel of a homomorphism $f(p, a): K[X] \rightarrow K(y[p, a])$. The set $y[p, a]$ is the union of all "hooks" corresponding to the nodes $(a(1, i), a(2, i))$ as $i$ ranges over $[1, p]$; more precisely,

$$
y[p, a]=\bigcup_{i=1}^{p}(\{a(1, i)\} \times[a(2, i), m(2)]) \cup([a(1, i), m(1)] \times\{a(2, i)\}) .
$$

Note that $y[p, E[p]]=y[p+1]$. The map $f(p, a)$ is defined to be identity on $y[p, a]$, and zero above the first hook, and in the region below the last hook or between any two hooks it is defined by expanding a minor of an appropriate size as described below. If $(i, j) \in[1, m(1)] \times[1, m(2)]$ is such that $i \geq a(1,1)$ and $j \geq a(2,1)$ and $(i, j) \notin y[p, a]$, then we can find a unique integer $u \in[1, p]$ which is largest with the property that $a(1, u)<i$ and $a(2, u)<j$. Now consider the $(u+1) \times(u+1)$ submatrix $M_{i j}(u)$ formed by the rows $a(1,1), \ldots, a(1, u), i$ and the columns $a(2,1), \ldots, a(2, u), j$ of $X$. Let $E[p, a, u]$ denote the bivector of length $u$ obtained by restricting $a$ to $[1,2] \times[1, u]$. We can find a unique polynomial $\widetilde{A}(X, p, a, i, j) \in K[y[p, a]]$ such that

$$
\operatorname{det} M_{i j}(u)=\operatorname{mor}(X, E[p, a, u]) X_{i j}-\widetilde{A}(X, p, a, i, j) .
$$

Now we define $f(p, a)$ to be the unique $K$-homomorphism of $K[X]$ into $K(y[p, a])$ such that

$$
f(p, a)\left(X_{i j}\right)= \begin{cases}0 & \text { if } i<a(1,1) \text { or } j<a(2,1) \\ X_{i j} & \text { if }(i, j) \in y[p, a] \\ \frac{\widetilde{A}(X, p, a, i, j)}{\operatorname{mor}(X, E[p, a, u])} & \text { if }(i, j) \notin y[p, a], i \geq a(1,1), \text { and } j \geq a(2,1) .\end{cases}
$$

The inclusion $I(p, a) \subseteq \operatorname{ker} f(p, a)$ is proved in an analogous manner, except that we now have more cases to consider. The proof of the desired equality can then be completed using (6.3) and by inducting on $p$. For details, see [A3].

From the definition of $f(p, a)$ it is clear that it induces a $K$-isomorphism of the quotient field of $K[X] / I(p, a)$ onto $K(y[p, a])$, and thus we can deduce the following ThEOREM (6.4). The quotient field of $K[X] / I(p, a)$ is a pure transcendental extension of $K$, and its transcendence degree is equal to $|y[p, a]|$. In other words, the irreducible projective variety $\mathcal{V}(p, a)$ defined by $I(p, a)$, is rational and its dimension is equal to $|y[p, a]|-1$.

Remark: Note that by the definition of $y[p, a]$, we clearly have

$$
|y[p, a]|=\sum_{i=1}^{p}[m(1)-a(1, i)+m(2)-a(2, i)+1]=p+\sum_{\hat{k}=1}^{2} \sum_{i=1}^{p}[m(\hat{k})-a(\hat{k}, i)]
$$

and thus the dimension of $\mathcal{V}(p, a)$ given by Theorem (6.4) is in agreement with Corollary (6.2), as is to be expected.

Now we proceed to show how indexed monomials can be used to give another set of bases for the homogeneous components of $I(p, a)$ and $K[X] / I(p, a)$. The ideal 
$I(p, a)$ being prime, cannot, in general, contain the usual monomials. So we must adjust them suitably. Let us explain how one can do this.

Given any bivector $b$ bounded by $m$, we associate to it a monomial, denoted by $d(b)$, which represents the product of the indeterminates on the principal diagonal of the submatrix of $X$ corresponding to $b$; thus

$$
X^{d(b)}=\prod_{i} X_{b(1, i) b(2, i)}
$$

where $i$ varies from 1 to the length of $b$. We also allow the bivector to have length 0 , in which case we set $X^{d(b)}=1$, i.e., $d(b)(y)=0$ for all $y \in[1, m(1)] \times[1, m(2)]$. Now let us fix some $V \in \mathbb{N}$. To a monomial $t \in \operatorname{mon}(2, m, V)$, we shall associate a bivector $b=g(t)$, and this will have the property that $X^{d(b)}$ divides $X^{t}$. The basis of indexed monomials for $K[X]_{V}$, which induces bases for $I(p, a)_{V}$ and $K[X]_{V} / I(p, a)_{V}$, is given by $\{w(t): t \in \operatorname{mon}(2, m, V)\}$ where

$$
w(t)=\frac{X^{t}}{X^{d(g(t))}} \operatorname{mor}(X, g(t)) .
$$

In order to define the associated bivector $b=g(t)$, we first order the elements of the rectangle lexicographically, i.e., for any $y=(y(1), y(2)), \tilde{y}=(\tilde{y}(1), \tilde{y}(2))$ in $[1, m(1)] \times[1, m(2)]$, we define

$$
y<\tilde{y} \Longleftrightarrow \text { either } y(1)<\tilde{y}(1) \text { or } y(1)=\tilde{y}(1) \text { and } y(2)<\tilde{y}(2) \text {. }
$$

And we put the reverse lexicographic order on monomials, i.e., for any $t, t^{*}$ in $\operatorname{mon}(2, m, V)$, we define

$$
t<t^{*} \Longleftrightarrow \exists y \text { such that } t(y)>t^{*}(y) \text { and } t(\tilde{y})=t^{*}(\tilde{y}) \forall \tilde{y}<y \text {; }
$$

here $y$ and $\tilde{y}$ denote elements of $[1, m(1)] \times[1, m(2)]$. Note that this gives a total order on $\operatorname{mon}(2, m, V)$, and that $\operatorname{mon}(2, m, V)$ is a finite set, so in particular, we may speak of the least element in any nonempty subset of $\operatorname{mon}(2, m, V)$. The associated bivector $b=g(t)$ can now be defined by considering the four cases below.

Case $0: \operatorname{ind}(t) \leq p, \operatorname{ind}\left(t_{1 i}\right)<i$, and $\operatorname{ind}\left(t_{2 j}\right)<j$ for all $i, j \in[1, p]$.

In this case we take $b=g(t)$ to be the empty bivector of length 0 so that $X^{d(b)}=1$.

Case 1: $\operatorname{ind}\left(t_{1 i}\right) \geq i$ for some $i \in[1, p]$.

In this case we let $u$ be the smallest such $i$ and $b=g(t)$ be the unique bivector of length $u$ such that its associated monomial $d(b)$ is the least among $d\left(b^{*}\right)$ where $b^{*}$ ranges over the nonempty set of all bivectors bounded by $m$ and of length $u$ such that $b^{*}(1, u)<a(1, u)$ and $X^{d\left(b^{*}\right)}$ divides $X^{t}$.

Case 2: $\operatorname{ind}\left(t_{1 i}\right)<i$ for all $i \in[1, p]$ and $\operatorname{ind}\left(t_{2 j}\right) \geq j$ for some $j \in[1, p]$.

In this case we let $u$ be the smallest such $j$ and $b=g(t)$ be defined exactly as in Case 1 except that the condition $b^{*}(1, u)<a(1, u)$ is replaced by $b^{*}(2, u)<a(2, u)$.

Case 3: $\operatorname{ind}\left(t_{1 i}\right)<i$, ind $\left(t_{2 j}\right)<j$ for all $i, j \in[1, p]$ and $\operatorname{ind}(t)>p$.

In this case $b=g(t)$ is defined to be the unique bivector of length $p+1$ such that $d(b)$ is the least among all $d\left(b^{*}\right)$ where $b^{*}$ ranges over the nonempty set of all bivectors bounded by $m$ and of length $p+1$ such that $X^{d\left(b^{*}\right)}$ divides $X^{t}$.

We now show that $\{w(t): t \in \operatorname{mon}(2, m, V)\}$ is a linearly independent subset of $K[X]$. To this end, we first note that for any bivector $b$ bounded by $m$, the associated monomial $d=d(b)$ is the least among all the monomials in the expansion of the minor of $X$ corresponding to $b$; in other words, we can write

$$
\operatorname{mor}(X, b)=X^{d}+\sum_{r>d} \epsilon_{r} X^{r} \quad \text { with } \epsilon_{r} \in\{0,1,-1\} \text {. }
$$

Consequently, for any $t \in \operatorname{mon}(2, m, V)$, we can write

$$
w(t)=X^{t}+\sum_{s>t} \epsilon_{s} X^{s} \quad \text { with } \epsilon_{s} \in\{0,1,-1\} .
$$


Using this we can deduce the following

Lemma (6.5). $\{w(t): t \in \operatorname{mon}(2, m, V)\}$ is a $K$-linearly independent subset of $K[X]$.

Proof: Suppose a nontrivial linear combination $\sum \alpha_{t} w(t)$ were zero, then upon letting $t^{*}=\min \left\{t: \alpha_{t} \neq 0\right\}$, by (20) we see that

$$
\alpha_{t^{*}} X^{t^{*}}+\sum_{s^{*}>t^{*}} \beta_{s^{*}} X^{s^{*}}=0 \quad \text { for some } \beta_{s^{*}} \in K,
$$

and hence $\alpha_{t^{*}}=0$, which is a contradiction.

Note that if $t \in \operatorname{mon}(2, m, V)$ is such that $t \notin \operatorname{mon}(2, m, p, a)$, then $\operatorname{mor}(X, g(t)) \in$ $I(p, a)$, and hence $w(t) \in I(p, a)$. In fact, this conclusion holds even when we restrict to subsets of the rectangle, which are "saturated" in the sense that if a principal diagonal of a minor is there, then so is the minor. More precisely, we make the following

Definition (6.6). A subset $Y$ of $[1, m(1)] \times[1, m(2)]$ is said to be saturated if for any bivector $b$ bounded by $m$, we have

$$
(b(1, i), b(2, i)) \in Y \forall i \Rightarrow(b(1, i), b(2, j)) \in Y \forall i, j
$$

where $i$ and $j$ vary from 1 to the length of $b$.

Given any subset $Y$ of $[1, m(1)] \times[1, m(2)]$, we let

$$
\begin{aligned}
& \operatorname{mon}(2, m ; Y)=\{t \in \operatorname{mon}(2, m): \operatorname{supp}(t) \subseteq Y\} \\
& \operatorname{mon}(2, m, V ; Y)=\operatorname{mon}(2, m, V) \cap \operatorname{mon}(2, m ; Y) \\
& \operatorname{mon}(2, m, p, a ; Y)=\operatorname{mon}(2, m, p, a) \cap \operatorname{mon}(2, m ; Y) \\
& \operatorname{mon}(2, m, p, a, V ; Y)=\operatorname{mon}(2, m, p, a, V) \cap \operatorname{mon}(2, m ; Y)
\end{aligned}
$$

and we let $I^{*}(p, a, Y)$ denote the ideal in $K[Y]$ generated by

$$
\{\operatorname{mor}(X, b): b \not \leq a\} \cap K[Y] .
$$

THEOREM (6.7). Let the notation be as above. We have the following

(i) $\{w(t): t \in \operatorname{mon}(2, m, V)\}$ is a $K$-basis of $K[X]_{V}$.

(ii) $\{w(t): t \in \operatorname{mon}(2, m, V) \backslash \operatorname{mon}(2, m, p, a, V)\}$ is a $K$-basis of $I(p, a)_{V}$, whereas $\{w(t): t \in \operatorname{mon}(2, m, p, a, V)\}$ is a $K$-basis of $K[X]_{V} / I(p, a)_{V}$.

(iii) For any saturated subset $Y$ of $[1, m(1)] \times[1, m(2)],\{w(t): t \in \operatorname{mon}(2, m, V ; Y)\}$ is a $K$-basis of $K[Y]_{V}$, and moreover, $\{w(t): t \in \operatorname{mon}(2, m, V ; Y) \backslash \operatorname{mon}(2, m, p, a, V ; Y)\}$ is a $K$-basis of $I^{*}(p, a, Y)_{V}$, whereas $\{w(t): t \in \operatorname{mon}(2, m, p, a, V ; Y)\}$ is a $K$-basis of $K[X]_{V} / I^{*}(p, a, Y)_{V}$.

Proof: Clearly $|\operatorname{mon}(2, m, V)|=\operatorname{dim}_{K} K[X]_{V}$, and hence (i) follows from (6.5). Next, by (5.2) and (6.1), we see that $|\operatorname{mon}(2, m, V) \backslash \operatorname{mon}(2, m, p, a, V)|=\operatorname{dim}_{K} I(p, a)_{V}$, and hence (6.5) implies the first part of (ii), whereas the second part of (ii) follows from (i) and the first part of (ii). Finally, since $Y$ is saturated, for any $t \in \operatorname{mon}(2, m, V ; Y), w(t) \in K[Y]_{V}$, and moreover if $t \notin \operatorname{mon}(2, m, p, a, V ; Y)$, then $w(t) \in I(p, a)_{V}$. Also we obviously have $|\operatorname{mon}(2, m, V ; Y)|=\operatorname{dim}_{K} K[Y]_{V}$. Hence (iii) can be deduced from (i) and (ii).

Corollary (6.8). For any saturated subset $Y$ of $[1, m(1)] \times[1, m(2)]$, we have $I^{*}(p, a, Y)=I(p, a) \cap K[Y]$, and hence the ideal $I^{*}(p, a, Y)$ is prime.

Remark: Note that a ladder shaped subset $L$ of $[1, m(1)] \times[1, m(2)]$ is clearly saturated, and as a special case of (6.8), it follows that the ideal $I_{p}(L)$ discussed in the Introduction is prime. Primality in this special case is also proved in $[\mathrm{N}]$.

\section{Some Recent Developments}

In this section, we shall briefly discuss some of the questions suggested by the work of Abhyankar that was described in the previous sections, and indicate the 
progress that has taken place in the last five years. Most of the problems mentioned here were posed in [A3].

7.1. Enumeration of Standard Multitableaux. Here one has the combinatorial problem of finding a nice polynomial formula for $|\operatorname{stab}(q, m, p, a, V)|$ where $q$ can be any positive integer. Recall that in Section 3, we obtained formulas $F^{(8)}$ and $F^{(7)}$ for this, and the formulas $F^{(6)}$ and $F^{(5 k)}$ in the case of even $q$. A polynomial formula was, however, obtained only when $q=2$, and we have already seen how crucial that was. An answer to this problem in the case of even $q$, was given in 1986 by Ghorpade (cf. [Gh1]). It turns out that the higher dimensional determinants introduced by Cayley in 1843 [C] appear naturally in such a formula. For definitions and basic properties of higher dimensional determinants, see the last chapter of $[\mathrm{MM}]$ or Section 4 of [Gh1]. One of the key steps in obtaining this formula is to prove the so called Multiproduct Lemmas which say that if $A_{1}, A_{2}, \ldots, A_{q}$ are any integers and $V$ is an indeterminate over $\mathbb{Q}$, then

$$
\begin{gathered}
\left(\begin{array}{c}
V \\
A_{1}
\end{array}\right)\left(\begin{array}{c}
V \\
A_{2}
\end{array}\right) \cdots\left(\begin{array}{c}
V \\
A_{q}
\end{array}\right)=\sum_{G=0}^{S_{q}} \phi_{G}\left(A_{1}, \ldots, A_{q}\right)\left(\begin{array}{c}
V \\
S_{q}-G
\end{array}\right) \\
{\left[\begin{array}{c}
V \\
A_{1}
\end{array}\right]\left[\begin{array}{c}
V \\
A_{2}
\end{array}\right] \cdots\left[\begin{array}{c}
V \\
A_{q}
\end{array}\right]=\sum_{G=0}^{S_{q}}(-1)^{G} \phi_{G}\left(A_{1}, \ldots, A_{q}\right)\left[\begin{array}{c}
V \\
S_{q}-G
\end{array}\right]}
\end{gathered}
$$

where for any $k \in[1, q], S_{k}=A_{1}+\cdots+A_{k}$, and for any $G \in \mathbb{Z}, \phi_{G}$ is the symmetric function of $A_{1}, \ldots, A_{q}$ defined by

$$
\phi_{G}\left(A_{1}, A_{2}, \ldots, A_{q}\right)=\sum_{\substack{e_{1}, \ldots, e_{q} \in \mathbb{Z} \\
e_{q}=G}} \prod_{k=1}^{q}\left(\begin{array}{c}
S_{k}-A_{k} \\
A_{k}
\end{array}\right)\left(\begin{array}{c}
A_{k} \\
e_{k}-e_{k-1}
\end{array}\right)
$$

with the convention that $e_{0}=0$. For details, see [Gh1]. It may be interesting to know whether a polynomial formula for $|\operatorname{stab}(q, m, p, a, V)|$ can be obtained in the case of odd $q$.

7.2. Straightening Law in Higher Dimensions. Now that we have the notion of higher dimensional determinant, to a multivector $b$ bounded by $m=(m(1), \ldots, m(q))$ and of width $q$, we can correspond a "multiminor" of an $m(1) \times m(2) \times \cdots \times m(q)$ matrix $X=\left(X_{i_{1} i_{2} \ldots i_{q}}\right)$ whose entries are indeterminates over a field $K$. A multitableau gives a monomial in multiminors, and we may ask whether the ones corresponding to standard multitableaux give a basis of $K[X]$. In $[\mathrm{AG}]$ it is proved that the "standard monomials in multiminors" are always linearly independent. However, as a consequence of the formula for $|\operatorname{stab}(q, m, p, a, V)|$, it can be seen that they do not, in general, form a basis, i.e., they are too few to generate $K[X]$. For details, see $[\mathrm{AG}]$ and [Gh1]. It may be interesting to find a basis for $K[X]$ which naturally extends this set. It may also be interesting to investigate the primality of the ideal in $K[X]$ generated by all $p \times p \times \cdots \times p$ minors of the multimatrix $X$.

\subsection{Bijection between Standard Bitableaux and Indexed Monomials.} From (5.8), we know that the sets $\operatorname{stab}(2, m, p, a, V)$ and $\operatorname{mon}(2, m, p, a, V)$ have the same cardinality, and so we may ask whether one can construct an explicit bijection between them. Using the correspondence given by G. Robinson, C. Schensted, and D. Knuth, Abhyankar and Kulkarni have obtained such a bijection. As a corollary, we get a bijective proof of the identity $|\operatorname{stab}(2, m, V)|=\operatorname{dim}_{K} K[X]_{V}$, which was proved in (3.7) using enumeration. For details, see [AK2]. A variation of this bijection which, roughly speaking, corresponds to using columns instead of rows, is described in [AK3]. 
7.4. Standard Multitableaux and Monomials. Let $X$ be a $q$-dimensional matrix as in 7.2. Analogous to $\operatorname{mon}(2, m, p, a, V)$, we can define the set $\operatorname{mon}(q, m, p, a, V)$ of monomials on a hypercube, i.e., in $K[X]$. From 7.2, we already know that the two sets $\operatorname{stab}(q, m, p, a, V)$ and $\operatorname{mon}(q, m, p, a, V)$ cannot, in general, have the same cardinality (since mon $(2, m, V)$ generates $\left.K[X]_{V}\right)$. Using the Robinson-SchenstedKnuth correspondence, or rather the Abhyankar-Kulkarni version of it, it is not difficult to see that for $q>1$, we can get an injection of $\operatorname{stab}(q, m, p, a, V)$ into $\operatorname{mon}(q, m, p, a, V)$ which is a surjection only if $q=2$ (and in some pathological cases). One way to do this could be to note that the bijection in 7.3 is valid even when the entries come from finite totally ordered sets $M_{1}$ and $M_{2}$ instead of [1, $m(1)$ ] and $[1, m(2)]$; now multitableaux become bitableaux with entries on one of its sides coming from a set of $(q-1)$-tuples, ordered suitably. And stab $(q, m, p, a, V)$ corresponds to a set of monomials on the hypercube, which is, in general, smaller than $\operatorname{mon}(q, m, p, a, V)$ when $q>2$. For other proofs of this, see [AJ1] and [AJ2]. It may be interesting to find an enumerative formula for $|\operatorname{mon}(q, m, p, a, V)|$ for any $q$.

7.5. Ladder Determinantal Varieties. We have already discussed these in the Introduction and in the Remark following (6.7). A problem concerning these is to find the Hilbert function of the ideal $I_{p}(L)$ of all $p \times p$ minors in a ladder shaped subset $L$ of the rectangle $[1, m(1)] \times[1, m(2)]$, or, more generally, of the ideal $I^{*}(p, a, Y)$ for any saturated subset $Y$ of the rectangle. Note that when $Y=[1, m(1)] \times[1, m(2)], F^{(3 k)}$ gives the Hilbert function of $I^{*}(p, a, Y)=I(p, a)$. For a ladder $L$, an explicit formula for the Hilbert function of $I_{p}(L)$, in the case of $p=2$, was obtained by Kulkarni in $1985[\mathrm{~K}]$. In 1987, Abhyankar and Kulkarni [AK1] showed that the ideal $I^{*}(p, a, Y)$ is Hilbertian, i.e., its Hilbert function is a polynomial for all nonnegative integers. However, there remained the problem of finding an explicit formula for the Hilbert function of $I_{p}(L)$ for any $p$. This has recently been solved, and for details we refer to [Gh2]. For the connection of determinantal and ladder determinantal ideals with Schubert varieties and such, see $[\mathrm{G}],[\mathrm{Mu}]$, [Mus1], [Mus2], [Se].

7.6. Coefficients of the Hilbert Function of $I(p, a)$. Recall that for any $k \in$ $[1,2]$,

$$
F^{(3 k)}(m, p, a, V)=\sum_{D \in \mathbb{Z}}(-1)^{D} F_{D}^{(3 k)}(m, p, a)\left[\begin{array}{c}
V \\
C(m, p, a)-D
\end{array}\right]
$$

gives the Hilbert function as well as the Hilbert polynomial of $I(p, a)$. The coefficients $F_{D}^{(3 k)}(m, p, a)$ have the interesting property that they are all nonnegative and the positive ones among them appear consecutively, i.e., $\left\{D \in \mathbb{Z}: F_{D}^{(3 k)}(m, p, a) \neq\right.$ $0\}$ is an interval of the type $\left[0, D^{*}\right]$, for some nonnegative integer $D^{*}$. In particular, $F_{0}^{(3 k)}(m, p, a)>0$, which follows indirectly from (6.2) or from the fact that $F_{D}^{(3 k)}=F_{D}^{(1 k)}$ and that the latter satisfy recurrence relations as in (5.1) [these recurrence relations also show that $F_{D}^{(3 k)}$ is always nonnegative]. Direct combinatorial proofs of the nonnegativity of $F_{D}^{(3 k)}$ and the positivity of $F_{0}^{(3 k)}$ were given by Udpikar; he also proved that $\left\{D \in \mathbb{Z}: F_{D}^{(3 k)}(m, p, a) \neq 0\right\}$ is an interval of the form $\left[0, D^{*}\right]$. For details, see $[\mathrm{U}]$.

\section{REFERENCES}

[A1] S. S. Abhyankar, Combinatoire des tableaux de Young, varietes determinantielles et calcul de fonctions de Hilbert, Rend. Sem. Mat. Univers. Politecn. Torino, 42 (1984), 65-88.

[A2] S. S. Abhyankar, Determinantal loci and enumerative combinatorics of Young tableaux, Algebraic Geometry and Commutative Algebra in Honor of Masayoshi Nagata, Volume 1, Kinokuniya Company Ltd., Tokyo (1987), 1-26. 
[A3] S. S. Abhyankar, Enumerative Combinatorics of Young Tableaux, Marcel Dekker, New York, 1988 .

[AG] S. S. Abhyankar and S. R. Ghorpade, Young tableaux and linear independence of standard monomials in multiminors of a multimatrix, to appear in Discrete Math.

[AJ1] S. S. Abhyankar and S. B. Joshi, Generalized rodeletive correspondence between multitableaux and multimonomials, preprint.

[AJ2] S. S. Abhyankar and S. B. Joshi, Generalized codeletion and standard multitableaux, Canadian Math. Soc. Conference Proc., Volume 10 (1989), 1-24.

[AK1] S. S. Abhyankar and D. M. Kulkarni, On hilbertian ideals, Linear Algebra and its Appl., 116 (1989), 53-79

[AK2] S. S. Abhyankar and D. M. Kulkarni, Bijection between indexed monomials and standard bitableaux, Discrete Math., 79 (1989/90) 1-48.

[AK3] S. S. Abhyankar and D. M. Kulkarni, Coinsertion and standard bitableaux, preprint.

[C] A. Cayley, On the theory of determinants, Trans. Camb. Phil. Soc., 8, (1843), 75-88.

[DEP] C. DeConcini, D. Eisenbud, and C. Procesi, Young diagrams and determinantal varieties, Inv. Math., 56 (1980), 129-165.

[DKR] J. Desarmenien, J. P. S. Kung, and G. C. Rota, Invariant theory, Young bitableaux, and combinatorics, Adv. in Math., 27 (1978), 63-92.

[DRS] P. Doubilet, G. C. Rota, and J. Stein, Foundations of Combinatorics IX: Combinatorial Methods in Invariant Theory, Studies in Appl. Math., 53 (1974), 185-216.

[G] A. Galigo, Computations of some Hilbert functions related with Schubert calculus, Algebraic Geometry, Sitges (Barcelona), Lecture Notes in Math. No. 1124, Springer-Verlag, New York, 1983.

[Gh1] S. R. Ghorpade, Young multitableaux and higher dimensional determinants, Adv. in Math., 121 (1996), 167-195.

[Gh2] S. R. Ghorpade, Hilbert functions of ladder determinantal varieties, Discrete Math. 246 (2002), 131-175. .

[Ho] W. V. D. Hodge, Some enumerative results in the theory of forms, Proc. Camb. Phil. Soc., 39 (1943), 22-30.

[HE] M. Hochster and J. A. Eagon, Cohen-Macaulay rings, Invariant theory, and the generic perfection of determinantal loci, Amer. J. Math., 93 (1971), 1020-1058.

[J] G. D. James, The Representation Theory of Symmetric Groups, Lecture Notes in Math. No. 682, Springer-Verlag, New York, 1977.

[K] D. M. Kulkarni, Thesis, Purdue Univ., 1985

$[\mathrm{Ku}] \quad$ J. P. S. Kung, Young Tableaux in Combinatorics, Invariant Theory, and Algebra, Academic Press, New York, 1982.

[M] K. R. Mount, A remark on determinantal loci, J. London Math. Soc., 42 (1967), 595-598.

[MM] T. Muir and W. Metzler, A Treatise on the Theory of Determinants, Longman's Green and Co., London, 1933.

[Mu] S. B. Mulay, Determinantal loci and the flag variety, Adv. in Math., 74 (1989), 1-30.

[Mus1] C. Musili, Some properties of Schubert varieties, J. Indian Math. Soc., 36 (1974), 131-145.

[Mus2] C. Musili, Applications of standard monomial theory, preprint.

[N] H. Narasimhan, Irreducibility of ladder determinantal varieties, J. of Algebra, 102 (1986), 162-185.

[P] E. Pascal, Sopra le relacioni che possono sussistere identicanemte fra formacioni simboliche del tipo invariantivo nella teoria generale delle forme algebriche, Mem. Accad. Lincei Roma, Series V, 4a (1888), 375-387.

[S] B. Sagan, The Symmetric Group: Representations, Combinatorial Algorithms, and Symmetric Functions, to appear in Wadsworth and Brooks/Cole, Monterey.

[Se] C. S. Seshadri, Introduction to Standard Monomial Theory, Brandeis Lecture Notes 4, 1985

[St] D. Stanton (ed.), Invariant theory and tableaux, Springer-Verlag, New York, 1990.

[U] S. G. Udpikar, On Hilbert polynomial of certain determinantal ideals, Internat. J. Math. \& Math. Sci., 14 (1991), 155-162.

[Y] A. Young, The Collected Papers of Alfred Young, Univ. of Toronto Press, 1977.

[ZS] O. Zariski and P. Samuel, Commutative Algebra, Vol. II, Van Nostrand, New York, 1960.

Department of Mathematics

Indian Institute of TeChNology Bombay

POWAi, Bombay 400076, India

E-mail address: srg@math.iitb.ac.in

$U R L:$ http://www.math.iitb.ac.in/ srg 